Interfaces and Free Boundaries 12 (2010), 463-496 DOI $10.4171 / \mathrm{IFB} / 242$

\title{
The thin film equation with backwards second order diffusion
}

\author{
AMY NOVICK-COHEN \\ Department of Mathematics, Technion-IIT, Haifa 32000, Israel \\ E-mail:amync@tx.technion.ac.il \\ ANDREY SHISHKOV \\ Institute of Applied Mathematics and Mechanics, 83114 Donetsk, Ukraine \\ E-mail: shishkov@iamm.ac.donetsk.ua
}

[Received 18 February 2009 and in revised form 27 September 2010]

Dedicated to Roberta Dal Passo (1956-2007)

\begin{abstract}
We focus on the thin film equation with lower order "backwards" diffusion which can describe, for example, the evolution of thin viscous films in the presence of gravity and thermo-capillary effects, or the thin film equation with a "porous media cutoff" of van der Waals forces. We treat in detail the equation

$$
u_{t}+\left\{u^{n}\left(u_{x x x}+v u^{m-n} u_{x}-A u^{M-n} u_{x}\right)\right\}_{x}=0,
$$

where $v= \pm 1, n>0, M>m$, and $A \geqslant 0$. Global existence of weak nonnegative solutions is proven when $m-n>-2$ and $A>0$ or $v=-1$, and when $-2<m-n<2, A=0, v=1$. From the weak solutions, we get strong entropy solutions under the additional constraint that $m-n>-3 / 2$ if $v=1$. A local energy estimate is obtained when $2 \leqslant n<3$ under some additional restrictions. Finite speed of propagation is proven when $m>n / 2$, for the case of "strong slippage", $0<n<2$, when $v=1$ based on local entropy estimates, and for the case of "weak slippage", $2 \leqslant n<3$, when $v= \pm 1$ based on local entropy and energy estimates.
\end{abstract}

2010 Mathematics Subject Classification: 35K65, 35K30, 35K35, 35G25, 76A20, 76D08.

Keywords: Thin film equation; backwards diffusion; higher order parabolic equations; degenerate parabolic equations; finite speed of propagation.

\section{Introduction}

The thin film equation [44]

$$
u_{t}+\left\{u^{n}\left(u_{x x x}\right)\right\}_{x}=0, \quad n>0,
$$

with $n=3$ models the dynamics of thin viscous films with no slip boundary conditions, and with $n=1$ it models Hele-Shaw flow. Often 1.1 needs to be augmented with various lower order terms in order to take into account the presence of additional physical effects, and certain such equations will be considered here. See [41] for a survey and review. Some of the physical systems which are accommodated by the analysis in this paper are listed below.

(i) The evolution of thin viscous films in the presence of gravity and thermo-capillary effects is modeled by

$$
u_{t}+\left\{u^{n}\left(u_{x x x}+u^{m-n} u_{x}-A u^{M-n} u_{x}\right)\right\}_{x}=0,
$$


where $m, n, M, A$ are constants such that $A \geqslant 0, n>0, m<M$, as well as the more accurate variant of $(1.2)$ given by

$$
u_{t}+\left\{u^{n}\left(u_{x x x}+h^{\prime}(u) u_{x}\right)\right\}_{x}=0,
$$

where $h^{\prime}(u)=-\bar{v} G+B_{1} /\left(u\left(1+B_{2} u\right)^{2}\right), G, B_{1}, B_{2}$ are positive constants, $\bar{v}= \pm 1$, and where $\bar{v}=+1[-1]$ represents stabilizing [destabilizing] gravitational forces [41, 49, 42]. Equation (1.2) with $A=0$,

$$
u_{t}+\left\{u^{n}\left(u_{x x x}+u^{m-n} u_{x}\right)\right\}_{x}=0,
$$

models thin films with thermo-capillary effects but without gravitational effects when $m-n=-1$ [21], or with destabilizing gravitational effects but without capillary effects when $m-n=0$. The value of $n$ in (1.2), 1.3 reflects the assumptions on the slip conditions at the interface of the thin film with the underlying substrate, with $n=3$ modeling no slip and $0<n<3$ modeling various types of slip.

(ii) The equation

$$
u_{t}+\left\{u^{n}\left(u_{x x x}+h^{\prime}(u) u_{x}\right)\right\}_{x}=0
$$

describes (a) the evolution of a thin viscous film in the presence of attractive polar forces if $h(u)=$ $-b_{1} e^{-u / b_{2}}$ where $b_{1}, b_{2}$ are positive constants, or (b) the evolution of a thin viscous film in the presence of attractive van der Waals forces if $h(u)=B u^{-b}$, where $B<0$ is a (negative) Hamaker constant and $b$ is a positive constant. More generally, if $\lim _{u \downarrow 0} h^{\prime}(u)>0[<0], h(u)$ is said to represent limiting attractive [repulsive] forces. Equations of the form (1.2), (1.4) can represent a combination of attractive and repulsive forces, and the limiting power $\lim _{u \downarrow 0} u h^{\prime \prime}(u) / h^{\prime}(u)$ can assume a range of both positive and negative values in modeling various forces such as polar forces, van der Waals forces, as well as "porous media cut-off" of van der Waals forces. See [38, 41, 11, 31, 16].

(iii) The Hocherman-Rosenau equation [29]

$$
\left.u_{t}+\left\{f(u) u_{x x x}+g(u) u_{x}\right)\right\}_{x}=0
$$

was proposed as a generalization of the cylindrical Kuramoto-Sivashinsky equation, which models low Reynolds number two phase cylindrical flows. Equation 1.5 with $f(u), g(u)>0$ has been used as a prototype equation for studying the relative strength of the second and fourth order terms in determining criteria for blow up [29, 14]. Setting $f(u)=u^{n}$ and $g(u)=u^{m}$ or $g=h^{\prime}(u)$ with $h^{\prime}(u)>0$ in 1.5 yields 1.4 with "limiting attractive forces".

To understand these various models, we shall focus on the equation

$$
u_{t}+\left\{u^{n}\left(u_{x x x}+v u^{m-n} u_{x}-A u^{M-n} u_{x}\right)\right\}_{x}=0,
$$

where $v= \pm 1$, and where $n, m, M, A$ are constants satisfying $A \geqslant 0, n>0, M>m$, with some further restrictions to be imposed later. All of the examples outlined above may be written in the form (1.6), except for (1.3) and (1.4)(a) in which

$$
h^{\prime}(u)=u(1+B u)^{-2} \quad \text { and } \quad h^{\prime}(u)=\frac{b_{1}}{b_{2}} e^{-u / b_{2}}
$$


respectively. Noting that

$$
\begin{gathered}
0<\frac{u}{(1+B u)^{2}}<\frac{1}{B^{2} u}, \\
0<\frac{b_{1}}{b_{2}} e^{-u / b_{2}}<\frac{b_{1}}{b_{2}},
\end{gathered}
$$

for $u \geqslant 0$, we see that the examples in 1.7 have upper bounds of the form $h^{\prime}(u)=v u^{m-n}-A u^{M-n}$ with $v=1, A=0$, and $m-n=-1$ and $m-n=0$ respectively, which facilitate the analysis of $(1.3)$ and $(1.4)$ (a). Our treatment of $(1.6)$ can be generalized to encompass (1.7) as well; some remarks in this direction are included.

In terms of the physical systems being modeled, it is reasonable to assume zero contact angle, $u_{x}=0$, and zero flux, $u^{n}\left(u_{x x x}+v u^{m-n} u_{x}-A u^{M-n} u_{x}\right)=0$, along the external boundaries of the domain, and thus

$$
u_{x}=u_{x x x}=0 \quad \text { if } u>0 \text { and } x= \pm a .
$$

While these boundary conditions are adopted here, other boundary conditions are possible. The zero contact angle condition reflects the physical assumption that the viscous liquid film completely wets the underlying substrate; non-zero contact angle conditions, while physical, have proven up to now to be difficult to implement in dynamical problem formulations [43]. A simple alternative to (1.10] is to impose periodic boundary conditions.

The term $\left\{u^{n}\left(v u^{m-n} u_{x}\right)\right\}_{x}$ with $v=+1$ in 1.6 is often referred to as a backwards diffusion term, since if one considers dynamics dominated by this term alone,

$$
u_{t}+\left\{u^{n}\left(u^{m-n} u_{x}\right)\right\}_{x}=0
$$

and one linearizes about a uniform positive state, then the resulting dynamics is given by the backwards (ill-posed) diffusion equation. Similarly, if $v=-1$, the term $\left\{u^{n}\left(v u^{m-n} u_{x}\right)\right\}_{x}$ in 1.6 is often referred to as forward diffusion, for obvious reasons [40]. We shall often refer to equation (1.6) with $v=+1$ as the unstable case and to equation (1.6) with $v=-1$ as the stable case, since in the context of thin films, 1.6 with $v=+1$ models limiting attractive forces which are destabilizing ("long wavelength unstable" in the terminology of [13]), and (1.6) with $v=-1$ models limiting repulsive forces which are stabilizing [41].

The degenerate Cahn-Hilliard equation

$$
u_{t}+\left\{u(1-u)\left[-\ln u+\ln (1-u)+\alpha u+\epsilon^{2} u_{x x}\right]_{x}\right\}_{x}=0
$$

whose solutions satisfy $0 \leqslant u \leqslant 1$ [23, 40], can also be said to be of the form (1.6) with $v=-1$, $n=1, m=0$ for $u$ near 0 or 1 . Equations similar to (1.4), (1.6) also arise in modeling structure formation and the dynamics of biofilms [33], as well as in modeling the dislocation density in plasticity theory [26].

Equation $(1.6)$ in the presence of forward or stabilizing diffusion

$$
u_{t}+\left\{u^{n}\left(u_{x x x}-u^{m-n} u_{x}\right)\right\}_{x}=0
$$

has been studied somewhat more thoroughly than the backwards or unstable variant

$$
u_{t}+\left\{u^{n}\left(u_{x x x}+u^{m-n} u_{x}\right)\right\}_{x}=0 .
$$

This is perhaps not surprising, as the behavior of the thin film equation changes qualitatively less upon adding a stabilizing term as opposed to adding a destabilizing term, and hence the analytical tools used in studying (1.1) may be more readily adapted to its analysis. 
Since (1.1) is designed to model the evolution of thin viscous films, nonnegative initial data should yield nonnegative solutions. Compactly supported initial data whose support may spread or shrink are also physically relevant. A pioneering step in the analysis of (1.1) was the proof of the existence of weak nonnegative solutions for $1<n<3$ by Bernis \& Friedman [7] for nonnegative $H^{1}$ initial data, which relied on energy and entropy estimates. The analysis in [7] was extended by Bernis [3] to the interval $0<n<3$. In [8], existence [nonexistence] of compactly supported spreading source type solutions to 1.1 was demonstrated for $0<n<3$ [ $n \geqslant 3$ ], and at the contact line, the solutions were seen to behave like $\left(x_{0}-x\right)^{2}$ for $0<n<3 / 2$ and like $\left(x_{0}-x\right)^{3 / n}$ for $3 / 2<n<3$. By developing refined entropy estimates, the existence of strong $\left(\mathcal{C}^{1}\right.$ for a.e. $\left.t>0\right)$ nonnegative solutions was demonstrated by Beretta, Bertsch \& Dal Passo [2] and by Bertozzi \& Pugh [12], and the solutions were seen to possess the regularity of the source type solutions at the contact line. Positivity properties were seen to depend strongly on the value of $n$ [7, 2], with "touchdown" being possible for small values of $n$ [9, 2]. Solutions were proven to become positive in finite time, and to converge to their mean as $t \rightarrow \infty$ [2, 12]. The only nonconstant steady states for (1.1) are of the form $(x-a)^{+}(b-x)^{+}$for $a<b$, which do not possess the regularity of the strong solutions [2, 12]. Finite speed of propagation of the support of strong solutions for $0<n<3$ was proven in [4, 5]. Some of the details of the various methodologies will be specfied further as we present our results. Since these basic studies, the analysis has been developed considerably to encompass, for example, the Cauchy problem for measured valued initial data [17], waiting time phenomena [19], higher dimensional studies of [1.1] [18, 15], as well as various numerical schemes [28, 27].

With regard to [1.12, existence of nonnegative distributional solutions was proven in [11] for $n>0$ and $0<m<1$. These solutions were shown to approach their mean as $t \rightarrow \infty$, becoming positive in finite time. Formal asymptotics and numerics were used to suggest the existence of advancing fronts for $n \geqslant 3$, as well as for $0<n<3$. Existence of nonnegative solutions for $\Omega \subset \mathbb{R}^{N}, N=1,2,3$, was then demonstrated for two special cases of 1.12 ; namely, for the model for defects in plasticity theory mentioned earlier [26], and for (1.11), the Cahn-Hilliard equation [23]. In [20], an existence theory for 11.12] was presented which encompassed the cases $n>1 / 8, m>-1, N \leqslant 3$ (with $n<4$ if $N=3$ ), with the case $0<n<1 / 8$ being treatable by adjusting the definition of the solution. The existence results in [11, 20] build on the results in [7, 2, 12], and in [20] the results build on [26] as well. In particular, use is made of augmented entropy estimates [2, 12], which indicate that the contact angle is zero for the solutions obtained if $0<n<3$ and $n-m>-2$. The constraint $n-m>-2$ allows the energy to be bounded from below for arbitrary nonnegative initial data $u_{0} \in H^{1}$. Regularity at the contact line was confirmed via formal asymptotic arguments in [11]. In [20], finite speed of propagation is demonstrated for $m>0,1 / 8<n<2$, and estimates are obtained for the speed of propagation; infinite speed of propagation is proven for $m<0,-2<m-n<-3 / 2$.

In [1], self-similar spreading source type solutions were shown to exist for 1.12 when $m-n=2$ and $0<n<3$, and not to exist if $n \geqslant 3$. The asymptotics of these solutions at the contact line was seen to match that of the source type solutions found for [1.1] in [8]. In terms of steady states, in [34] nonconstant positive periodic steady states and zero contact angle steady states are shown not to exist, though touchdown steady states with nonzero contact angle are shown to exist if $m-n>-2$.

In [28, 27], existence of positive solutions from nonnegative initial data was demonstrated via construction of a numerical scheme for (1.6) with $v=-1$ and $m-n<-2$. The inclusion of the term $-A u^{M}$ in 1.6 can be expected to enhance the rate of decay of the solution to its mean, and should not have any major effect on contact angle and propagation properties. In our analysis in the present paper, we closed gaps in the parameter range for $(1.12)$ and $(1.6)$ with $v=-1$, both in 
terms of existence and the finite speed of propagation property. Moreover, local energy and entropy estimates are obtained.

With regard to (1.13), less attention has been paid to the various existence, regularity, and finite speed of propagation properties for (1.13) than for (1.12), but there has been longstanding interest in the qualitative predictions of [1.13, in particular in regard to the possibilities of rupture [44, 50] and blow up [29]. There has been considerable interest in various self-similar and steady state solutions for [1.13, and their stability [46].

In terms of existence, regularity, and finite speed of propagation properties for 1.13 , the results up to now may be summarized as follows. In [13] it is demonstrated that if $0<n<3$ and $n \leqslant m<$ $n+2$, then there exist globally bounded nonnegative weak solutions to 1.13 which possess the finite speed of propagation (FSP) property, and have the same regularity at the contact line as was found in [8] for (1.1). We remark that formal asymptotics developed in [11] for (1.12) are equally valid for [1.13). The methodology in [13] relies on [7, 2, 12, 11], incorporating a "disjoining pressure" potential arising from the lower order terms [31] into the energy used in the basic energy estimate. Numerical evidence is given that blow up may occur if $m \geqslant n+2$. The case $n=1, m \geqslant 3=n+2$ is considered in [14], and it is demonstrated that for compactly supported nonnegative initial data whose initial energy is negative, there exists a solution which blows up in finite time. For the critical case, $n=1, m=3$, this implies blow up if the initial mass is sufficiently large [52]. It was conjectured in [13, 14] that $m-n \geqslant 0$ constitutes a necessary condition for well posed dynamics. The existence proof in [27], mentioned earlier in the context of $[1.12$, includes the case $v=1$, i.e. (1.13) with $n>0$ and $m<n-1$, for positive initial data. The inclusion of a term of the form $-A u^{M}$ in 1.6 when $v=1$ should eliminate the possibility of blow up, and should not have any major effect on contact angle and propagation properties.

The set of steady states and self-similar solutions is much richer for 1.13 than for 1.12 . For (1.13), positive periodic steady states exist, as do compactly supported "touchdown" steady state solutions with zero as well as with nonzero contact angles [34]. A study of steady states and stability based on energy criteria was undertaken in [36] for $m-n \in[1,2)$. It has been demonstrated [34, 35, 37. 46] that steady states with zero contact angle exist for all $0<n<3, n \leqslant m$; they are stable if $m \leqslant n+2,0<n \leqslant 2$, marginally stable if $m \leqslant n+2,2<n<3$, and unstable otherwise. In [10] for $v=1, n=3, m=-1$, steady states are seen to converge to a $\delta$-distribution in the limit in which repulsive forces are neglected; these results should perhaps be compared with the non-single-valued profiles seen in [38] which result when the term $u_{x x}$ is replaced by the mean curvature, a correction which becomes important in the singular limit. In terms of self-similar solutions, both spreading and blow up self-similar solutions are possible. Rupture self-similar solutions have also been observed and studied [53, 51]. If $m=n+2$, there exist spreading self-similar solutions if $0<n<3$, and none if $n \geqslant 3$ [1]. Blow up self-similar solutions were considered in [47], with existence being demonstrated for $0<n<3 / 2$ and nonexistence for $n \geqslant 3 / 2$. For a discussion of the stability of self-similar solutions, see [52, 46].

The focus of the present paper, however, is not on blow up, steady state solutions, or selfsimilar behavior, but rather on conditions that guarantee existence, regularity, and finite speed of propagation, completing and enhancing what was previously known, in order to construct a framework to understand, for example, the transition between rupture, positivity and touchdown properties, global existence, and blow up. Roughly speaking, with regard to existence for $v=-1$, $0<n<3$, there has been a gap for $n-2 \leqslant m \leqslant-1$; and now we have existence of both weak and strong solutions for $n-2<m \leqslant-1$, as well as for $n-2=m$ for a constrained set of initial conditions. For $v=1,0<n<3$, previous existence results have required $m \geqslant n$, and now we 
have existence of weak solutions in the interval $n-2<m<n$, as well as strong energy/entropy solutions in the subinterval $n-3 / 2<m<n$. Note also that for $v= \pm 1,0<n<3$, in the interval $n-2<m \leqslant n-1$, we do not require that the initial data be strictly positive [10]. In terms of FSP, for $v=-1,0<n<3$, there has been a gap at $0<n \leqslant 1 / 8$ which is now filled for $0<m<n+2$, as well as a gap at $2 \leqslant n<3$, which is now filled for $n / 2<m<n$. For $v=1,0<n<3$, there has been a gap at $0<m<n$, which is now filled for $n / 2<m<n$.

What physics pertains to the interval $v=-1,0<n<3, n-2 \leqslant m \leqslant-1$ or $v=1$, $0<n<3, n-2 \leqslant m<n$ ? The thin film equation with $v=1,0<n<3,-2<m-n<0$ can reflect a "porous media" cutoff of attractive (or repulsive if $v=-1$ ) van der Waals forces [41], thin films under the influence of thermo-capillary effects with $n=2$ or $3, m-n=-1, v=1$, [21] (with other values of $n, 0<n<3$, also possible), or a restricted Hocherman-Rosenau equation [29]. If one takes $v=1, n=2$ or $3, m \leqslant-1$, which can model, for example, the effects of a Lennard-Jones potential or a thin film on a layered solid substrate in the limit in which the limiting repulsive forces are neglected, the dynamics are known to lead to rupture [50, 53, 51].

As a first step, the existence of weak nonnegative solutions (see Definition 11) is demonstrated in $§ 2$. This is accomplished by means of the basic energy estimate [7, 2, 12]

$$
\sup _{0 \leqslant t \leqslant T} \int_{\Omega} u_{x}^{2} \mathrm{~d} x+\int_{0}^{T} \int_{\Omega} u^{n}\left(u_{x x x}+v u^{m-n} u_{x}-A u^{M-n} u_{x}\right)^{2} \mathrm{~d} x \mathrm{~d} t \leqslant C,
$$

where $\Omega=(-a, a), a \in(0, \infty)$ is arbitrary, $C$ depends only on the problem parameters and the initial conditions, and $0<T<\infty$. The estimate 1.14 holds for $n>0, m-n>-2$, $m<M$, with the additional constraint that $m-n<2$ if $v=1$ and $A=0$. Additionally, an entropy estimate [7, 2, 12] is obtained, using Gronwall's inequality for regularized solutions. The use here of Gronwall's inequality, which explicitly depends on the regularization parameter, differs somewhat from elsewhere [28], allowing us to control the lower order, possibly singular, forcing terms. These estimates, together with mass conservation,

$$
\int_{\Omega} u(x, t) \mathrm{d} x=\int_{\Omega} u(x, 0) \mathrm{d} x,
$$

imply global bounds from which existence of weak nonnegative solutions can be concluded, based on uniform Hölder continuity and continuation arguments. See Bernis \& Friedman [7] and Giacomelli [24].

To obtain the existence of a strong $\left(\mathcal{C}^{1}(\Omega)\right.$ for a.e. $\left.t>0\right)$ solution, a local entropy estimate is derived in $\S 3$, following [2]. For $v=1$, the additional constraint $m-n>-3 / 2$ is imposed and the local entropy estimate may be written as

$$
\begin{gathered}
\frac{1}{\alpha(\alpha+1)} \int_{\Omega} \zeta^{4} u^{1+\alpha}(x, T) \mathrm{d} x+A \int_{Q_{T}} \zeta^{4} u^{\alpha+M-1} u_{x}^{2} \mathrm{~d} x \mathrm{~d} t \\
\quad+c_{1}\left[\int_{P} \zeta^{4} u^{\alpha+n-2 \gamma+1}\left(u^{\gamma}\right)_{x x}^{2} \mathrm{~d} x \mathrm{~d} t+\int_{Q_{T}} \zeta^{4} u^{\alpha+n-3} u_{x}^{4} \mathrm{~d} x \mathrm{~d} t\right] \\
\leqslant \\
c_{2} \int_{Q_{T}}\left(\left|\zeta_{x}\right|^{4}+\left|\zeta \zeta_{x x}\right|^{2}\right) u^{n+\alpha+1} \mathrm{~d} x d t+c_{3} \int_{Q_{T}}\left|\left(\zeta^{3} \zeta_{x}\right)_{x}\right| u^{\alpha+m+1} \mathrm{~d} x \mathrm{~d} t \\
\quad+c_{4} \int_{Q_{T}} \zeta^{4} u^{\alpha+2 m-n+1} \mathrm{~d} x \mathrm{~d} t+\frac{1}{\alpha(\alpha+1)} \int_{\Omega} \zeta^{4} u_{0}{ }^{\alpha+1} \mathrm{~d} x
\end{gathered}
$$


where $Q_{T}=\Omega \times(0, T), P=\overline{\Omega \times(0, \infty)} \backslash\{u=0$ or $t=0\}$, and which holds for certain $\alpha \in(\max \{-2 m+n-1,-m-1\}, 2-n) \backslash\{0,-1\}$ and for $\gamma$ satisfying $(3.8)$. For $v=-1$, a similar estimate is obtained without additional restrictions. For both $v= \pm 1$, the local entropy estimate implies the global entropy estimate

$$
c_{5} \int_{Q_{T}} u^{\alpha+n-3} u_{x}^{4} \mathrm{~d} x \mathrm{~d} t+c_{6} \int_{Q_{T}} u^{\alpha+n-1} u_{x x}^{2} \mathrm{~d} x \mathrm{~d} t \leqslant c_{7} T, \quad T>0,
$$

which also holds for (1.1), and which implies strong solutions and certain positivity properties [2, 12]. We also present a refinement of Theorem 3.1 from [2] (see Corollary 3.4 in §3), which clarifies the set of $\beta$ for which $\mathcal{C}^{1}([-a, a])$ regularity for almost every $t>0$ is implied for $u^{1 / \beta}(\cdot, t)$, based on the local entropy estimates and the properties of the initial data. As in [25], a local energy estimate is derived for $2 \leqslant n<3$, under the additional restrictions that $m>(2 n-2) / 3$ if $2 \leqslant n<5 / 2$ and $m>n-3 / 2$ if $5 / 2 \leqslant n<3$.

In $\S 4$ and $\S 5$, we investigate conditions for the finite speed of propagation property (FSP) for (1.1). Two different techniques have been developed for studying the FSP property; both rely on energy and entropy estimates. The first method was developed by F. Bernis [4, 5] for the standard thin film equation, (1.1). For $0<n<2$, Bernis [4] introduced the weighted integral entropy function

$$
E_{T}(r):=\int_{0}^{T} \int_{-r}^{r}(r-|x|)^{4}\left(u^{(\alpha+n+1) / 2}\right)_{x x}^{2} \mathrm{~d} x \mathrm{~d} t, \quad 0 \leqslant r<r_{0}, T>0,
$$

for $0<r_{0}<a$, which corresponds to the third term on the left hand side of 1.16$)$ with $\zeta(x)=$ $(r-|x|)_{+}$. By taking $\operatorname{supp} u_{0} \subset \Omega \backslash\left\{|x|<r_{0}\right\}$ and deriving a nonlinear ordinary fourth order differential inequality for $E_{T}(r)$, we will demonstrate the FSP property. For $2 \leqslant n<3$, Bernis demonstrated the FSP property in [5] based on a similar ordinary differential inequality for an appropriately defined weighted integral energy function.

An alternative method for studying propagation properties for various thin-film-like equations was proposed for the case $0<n<2$ in [32] (see also the references and comments in [30]). This method relies on functional rather than differential inequalities for various entropy and energy functions. The emphasis in this technique is on cut-off functions rather than on weight functions, which lead to functional dependence on parameterized subdomains of $\Omega$. The main feature of the inequalities which are derived is that the minimal power of $u(x, t)$ in the terms on the left hand side of the inequality is strictly less than the minimal power of $u(x, t)$ which appears in the terms on the right hand side. Such inequalities in conjunction with the functional Stampacchia Lemma (see Lemma 6.1) and its generalization for systems of functional inequalities (see Lemma 6.2) can be used to demonstrate the FSP property in various contexts. In the present paper, we shall rely primarily on this second method.

This latter method (the FI-method) has been used [30] to obtain sharp estimates on the speed of propagation of the support of solutions to equation (1.1) with arbitrary $u_{0} \in L_{1}(\Omega)$, $\Omega \backslash \operatorname{supp} u_{0} \neq \varnothing$. In [20], using this method for the thin film equation with forward diffusion (1.12) with source type initial data, $m_{\text {crit }}=n+2$ was shown to be a transitional value between the long and short time asymptotics of the Barenblatt porous media behavior and the predicted behavior of source type solutions for the standard thin film equation [8]. Using the FI-method, sharp sufficient conditions were obtained in [19] relating the flatness at the edge of the support of $u_{0}$ to the phenomenon of waiting time for propagation of the support of solutions. Moreover for 
equation (1.1) with an additional nonlinear absorption term, in [45] a sharp sufficient condition on the flatness of $u_{0}$ guaranteeing the onset of shrinkage of the support was obtained, as well as estimates from below on the shrinkage rate. In future work, we hope to undertake similar analyzes for (1.6) with $v=1$.

For (1.6) with forward diffusion (with $A=0$ ), conditions for FSP as well as sharp estimates for the speed of propagation were obtained in [20] for the strong slippage case $(0<n<2)$ only. The results which we obtain here are for (1.6) with $v=-1$, with $2<n<3$, as well as for the much more delicate case of backward diffusion, $v=1$, where the lower order diffusion term "encourages" the destruction of the FSP property at all values of $m$. Our analysis makes use of some ideas from [25]. The proof given in $\S 4$ is for the strong slippage case in which $0<n<2$, and requires that $m>n / 2$ if $v=1$. It relies on the local entropy estimate from $\S 3$ for $\alpha$ positive and the Stampacchia Lemma for systems. We demonstrate that if supp $u_{0} \subset\{x \leqslant 0\}$, then there exists a continuous function $s(t)$ satisfying $s(0)=0$, and a positive time $T_{0}$ such that $\operatorname{supp} u(\cdot, t) \subset[-a, s(t)]$, $s(t)<a$ for all $t<T_{0}$, and $s\left(T_{0}\right)=a$. The proof in $\$ 5$ is for the weak slippage case in which $2<n<3$, and requires that $m>n / 2$. It is based on combining local entropy estimates for $-1<\alpha<0$ with the local energy estimates from $\S 3$, and again makes use of the Stampacchia Lemma for systems. We conjecture that the restriction $m>n / 2$ for $v=1$ is sharp or close to sharp. Note that in the stable case $v=-1$, the value $m=n / 3$ is critical in the context of asymptotics near the edge of traveling wave solutions with constant speed of propagation (see [11]). Since the asymptotics in [11] are also valid for the unstable case $v=1$, we expect that the FSP property fails in the unstable case for some $m$ near to $n / 3$. Thus we suspect that for $v=1$, the restriction $m>n / 2$ is sharp or close to sharp.

As to further questions and future directions, a question of interest in the present context is to understand exactly how strong the backward diffusion can become while maintaining something of the smoothing properties of the thin film equation with $n>0$. Thus one would like to identify transitional values in terms of rupture, positivity, touchdown, and infinite as opposed to finite spreading rates. Finally, one would like to extend all aspects of the analysis to higher dimensions.

The outline of the paper is as follows. The existence of weak nonnegative solutions is proven in $\S 2$. Existence of strong energy-entropy solutions is demonstrated in $\S 3$. Finite speed of propagation is proven in $\S 4$ for the case of weak slippage and in $\S 5$ for the case of strong slippage.

\section{Weak solutions}

In this section, we follow Bernis \& Friedman [7], relying on local parabolic regularity theory [22, 24] to obtain global existence of weak solutions, defined below. We regularize the initial data and use Gronwall's inequality in the context of the entropy estimate. This allows us to avoid regularizing the lower order terms [13, 14], and to widen the range of validity of the results.

Notation. Let $\Omega=(-a, a)$ where $a \in(0, \infty)$ is arbitrary, $Q_{T}=\Omega \times(0, T), 0<T<\infty$, and set $P_{T}=\overline{Q_{T}} \backslash\{u=0$ or $t=0\}, Q=Q_{\infty}, P=P_{\infty}$.

Let us consider the problem

$$
(\mathbb{P})\left\{\begin{array}{l}
u_{t}+\left(u^{n}\left(u_{x x x}+v u^{m-n} u_{x}-A u^{M-n} u_{x}\right)\right)_{x}=0, \quad(x, t) \in Q \\
u_{x}( \pm a, t)=u_{x x x}( \pm a, t)=0 \quad \text { when } u( \pm a, t) \neq 0, \quad t \in(0, \infty), \\
u(x, 0)=u_{0}(x), \quad x \in \bar{\Omega}
\end{array}\right.
$$


where $v= \pm 1$. We shall assume that the initial conditions satisfy

$$
u_{0} \in H^{1}(\Omega), \quad u_{0} \geqslant 0, \quad u_{0} \not \equiv 0 .
$$

While we shall look for solutions on a finite interval, we can always consider the parallel Cauchy and periodic problems obtained by extending the initial conditions via periodicity and reflection. This will allow us, for example, to directly implement the generalized Bernis inequalities for nonnegative periodic functions [25, Lemma B.1] in obtaining local energy estimates in $§ 3$. See also the remarks in [2, 14].

Definition 1 A function $u \in \mathcal{C}^{0,1 / 2,1 / 8}(\bar{\Omega} \times[0, \infty)) \cap L^{\infty}\left([0, \infty) ; H^{1}(\Omega)\right)$ is said to be a weak solution of $(\mathbb{P})$ if:

(a) $u \in \mathcal{C}^{4,1}(P), u \geqslant 0$,

(b) $u_{x}(x, t)=u_{x x x}(x, t)=0$ when $u(x, t) \neq 0$, for $(x, t) \in \partial \Omega \times(0, \infty)$,

(c) $J \equiv u^{n}\left(u_{x x x}+v u^{m-n} u_{x}-A u^{M-n} u_{x}\right) \in L^{2}(P)$,

(d) for all $\phi \in \operatorname{Lip}(\bar{\Omega} \times(0, \infty))$ with compact support, $u$ satisfies

$$
\int_{Q} u \phi_{t} \mathrm{~d} x \mathrm{~d} t+\int_{P} u^{n}\left(u_{x x x}+v u^{m-n} u_{x}-A u^{M-n} u_{x}\right) \phi_{x} \mathrm{~d} x \mathrm{~d} t=0,
$$

(e) $u(x, 0)=u_{0}(x)$ for $x \in \bar{\Omega}$.

Given this definition, we formulate

THEOREM 1 For the following range of parameter values:

(i) $v=-1, n>0, A \geqslant 0, n-2<m<M$,

(ii) $v=1, n>0, A>0, n-2<m<M$,

(iii) $v=1, n>0, A=0, n-2<m<n+2$,

there exists a solution to $(\mathbb{P})$ in the sense of Definition 1 for $u_{0}$ satisfying (2.1).

We shall find a weak solution to $(\mathbb{P})$ as the limit of a subsequence of smooth positive solutions to a regularized problem, with regularized initial conditions $u_{0 \epsilon}$. We shall require that for $\epsilon>0$ and for some $\lambda \in(0,1), \theta \in(0,2 / 5], u_{0 \epsilon}$ satisfies

$$
\begin{aligned}
& u_{0 \epsilon} \in \mathcal{C}^{4, \lambda}(\bar{\Omega}), \quad u_{0 \epsilon}^{\prime}( \pm a)=u_{0 \epsilon}^{\prime \prime \prime}( \pm a)=0, \quad u_{0}+\epsilon^{\theta} \leqslant u_{0 \epsilon} \leqslant u_{0}+1, \\
& u_{0 \epsilon} \rightarrow u_{0} \quad \text { in } H^{1}((-a, a)) \text { as } \epsilon \rightarrow 0 .
\end{aligned}
$$

Following [7], we set

$$
f_{\epsilon}(s)=\frac{|s|^{n+4}}{\epsilon|s|^{n}+s^{4}}
$$

when $0<s \ll 1, f_{\epsilon}(s) \approx s^{4} / \epsilon$ if $0<n<4$ and $f_{\epsilon}(s) \approx|s|^{n}$ if $n \geqslant 4$, which will allow us to guarantee the positivity of the approximants $u_{\epsilon}$ for $t>0$. Here and in the section which follows, $c_{i}, d_{i}$ denote positive constants that are independent of $\epsilon$, and $C_{i}(t)$ denotes a positive increasing function defined on $(0, \infty)$ that is independent of $\epsilon ; c_{i}, d_{i}, C_{i}(t)$ may depend on $\Omega, u_{0}$, and the problem parameters, and their value may change from line to line. 
Proof of Theorem 1 Let us define the approximating problem to be

$$
\left(\mathbb{P}_{\epsilon}\right) \begin{cases}u_{t}+\left\{f_{\epsilon}(u)\left(u_{x x x}+v u^{m-n} u_{x}-A u^{M-n} u_{x}\right)\right\}_{x}=0, & x \in \Omega, t>0, \\ u_{x}( \pm a, t)=u_{x x x}( \pm a, t)=0, & t>0, \\ u(x, 0)=u_{0 \epsilon}(x), & x \in \bar{\Omega}\end{cases}
$$

Problem $\left(\mathbb{P}_{\epsilon}\right)$ has a unique maximal positive solution, $u_{\epsilon}$, such that $u_{\epsilon} \in \mathcal{C}^{4, \lambda, \lambda / 4}\left(\bar{\Omega} \times\left[0, \tau_{\epsilon}\right)\right)$, $\tau_{\epsilon}>0$ (see [22, Theorem 6.3, p. 302] as well as the remark following the proof given there). That the associated Cauchy problem with periodically reflected initial data maintains the periodicity and reflection properties, can be seen by translating and reflecting the solution, then invoking uniqueness of the solutions to the Cauchy problem.

Testing $\left(\mathbb{P}_{\epsilon}\right)$ with $\phi \equiv 1$ and recalling 2.1, , 2.3, it follows that

$$
0<\overline{u_{\epsilon}}(t)=\overline{u_{0 \epsilon}} \leqslant \overline{u_{0}}+1, \quad t \in(0, T),
$$

where $\bar{v}:=|\Omega|^{-1} \int_{\Omega} v$. The equality $\overline{u_{\epsilon}}(t)=\overline{u_{0 \epsilon}}$ in 2.5 expresses mass conservation. Note that 2.5) also holds for solutions to similarly defined approximating problems for (1.3) and (1.4)(a).

Setting

$$
h(s)= \begin{cases}\frac{v s^{m-n+1}}{m-n+1}-A \frac{s^{M-n+1}}{M-n+1}, & m, M \neq n-1, \\ v \ln s-A \frac{s^{M-n+1}}{M-n+1}, & m=n-1, \\ \frac{v s^{m-n+1}}{m-n+1}-A \ln s, & M=n-1,\end{cases}
$$

and testing $\left(\mathbb{P}_{\epsilon}\right)$ with $-u_{\epsilon x x}-h\left(u_{\epsilon}\right)$, we obtain the energy estimate [7, 23, 31]

$$
\begin{aligned}
\left.\int_{\Omega}\left[\frac{1}{2} u_{\epsilon x}^{2}-H\left(u_{\epsilon}\right)\right]\right|_{t=T} \mathrm{~d} x+\int_{Q_{T}} f_{\epsilon}\left(u_{\epsilon}\right)\left(u_{\epsilon x x x}+h^{\prime}\left(u_{\epsilon}\right) u_{\epsilon x}\right)^{2} \mathrm{~d} x \mathrm{~d} t & \\
& =\int_{\Omega}\left[\frac{1}{2} u_{0 \epsilon x}^{2}-H\left(u_{0 \epsilon}\right)\right] \mathrm{d} x
\end{aligned}
$$

for $0<T<\tau_{\epsilon}$, where

$$
H(s)= \begin{cases}\frac{v s^{m-n+2}}{(m-n+2)(m-n+1)}-\frac{A s^{M-n+2}}{(M-n+2)(M-n+1)}, & m \neq M \neq n-1, \\ v(s \ln s-s)-\frac{A s^{M-n+2}}{(M-n+2)(M-n+1)}, & m=n-1, \\ \frac{v s^{m-n+2}}{(m-n+2)(m-n+1)}-A(s \ln s-s), & M=n-1 .\end{cases}
$$

Let $v=1$ and $-1 \leqslant m-n<2$. Then noting that

$$
-1 \leqslant s \ln s-s<s^{2}, \quad s>0,
$$

recalling the Gagliardo-Nirenberg inequality

$$
\|u\|_{L^{p}(\Omega)} \leqslant c_{1}\left\|u_{x}\right\|_{L^{2}(\Omega)}^{1 / 2}\|u\|_{L^{1}(\Omega)}^{1 / 2}+c_{2}\|u\|_{L^{1}(\Omega)}, \quad 1<p<4,
$$


and that the approximating solutions are positive and satisfy $(2.5)$, it follows that

$$
\int_{\Omega} H\left(u_{\epsilon}\right) \mathrm{d} x \leqslant \frac{1}{4} \int_{\Omega} u_{\epsilon x}^{2} \mathrm{~d} x+c_{3}\left(\int_{\Omega} u_{\epsilon} \mathrm{d} x\right)^{c_{4}}=\frac{1}{4} \int_{\Omega} u_{\epsilon x}^{2} \mathrm{~d} x+c_{5} .
$$

Suppose the restrictions on the parameters stated in Theorem 1 hold and that $-2<m-n<-1$ if $v=1$. Then recalling (2.7), and noting that for $A_{1}, A_{2}>0,0<\alpha<\beta$,

$$
A_{1} s^{\alpha}-A_{2} s^{\beta}<A_{1}\left(\frac{A_{1} \alpha}{A_{2} \beta}\right)^{\alpha /(\beta-\alpha)}, \quad s>0,
$$

and that by Hölder's inequality, if $0<\alpha<1$, then

$$
\int_{\Omega}|u|^{\alpha} \mathrm{d} x \leqslant \alpha \int_{\Omega}|u| \mathrm{d} x+(1-\alpha)|\Omega|,
$$

we obtain

$$
\int_{\Omega} H\left(u_{\epsilon}\right) \mathrm{d} x \leqslant c_{6}\left(\overline{u_{0}}+1\right)=c_{7} .
$$

From 2.6, 2.8, 2.9, we may now conclude that

$$
\frac{1}{4} \int_{\Omega} u_{\epsilon x}^{2}(x, T) \mathrm{d} x+\int_{Q_{T}} f_{\epsilon}\left(u_{\epsilon}\right)\left(u_{\epsilon x x x}+v u_{\epsilon}^{m-n} u_{\epsilon x}-A u_{\epsilon}^{M-n} u_{\epsilon x}\right)^{2} \mathrm{~d} x \mathrm{~d} t \leqslant c_{8} .
$$

REMARK 2.1 The constraint that $m-n<2$ when $v=1, A=0$ has been imposed to guarantee global existence. If $v=1, A=0$, and $m-n=2$, 2.10 remains valid if $\overline{u_{0}}$ is sufficiently small, and global existence again follows. See [13].

From 2.5, 2.10, we obtain

$$
\left\|u_{\epsilon}\right\|_{L^{\infty}\left(0, T ; H^{1}(\Omega)\right)} \leqslant c_{8}, \quad\left\|f_{\epsilon}^{1 / 2}\left(u_{\epsilon x x}+h\left(u_{\epsilon}\right)\right)_{x}\right\|_{L^{2}\left(Q_{T}\right)} \leqslant c_{9} .
$$

The following argument from [7] is by now standard. Noting that $u_{t}=-J_{x}, 2.11$ implies that

$$
\left\|u_{t}\right\|_{L^{2}\left(0, T ; H^{-1}(\Omega)\right)},\|J\|_{L^{2}\left(Q_{T}\right)} \leqslant c_{10},
$$

and the estimates 2.11), 2.12) can be seen to imply the uniform Hölder estimate

$$
\left\|u_{\epsilon}\right\|_{\mathcal{C}^{0,1 / 2,1 / 8}\left(\overline{Q_{T}}\right)} \leqslant c_{11} .
$$

See [7] for details.

REMARK 2.2 It is also possible to implement the above discussion when $u^{n}$ is replaced by $f(u)$ in $(\mathbb{P})$, for $f \in \mathcal{C}\left((0, \infty), \mathbb{R}^{+}\right)$, and to work with (see [7])

$$
f_{\epsilon}(u)=\frac{f(u) u^{4}}{\epsilon f(u)+u^{4}},
$$

under suitable assumptions on $f(u)$. 
We now demonstrate roughly as in [7, 24] that $u_{\epsilon} \geqslant 4 \sigma>0$ in $\bar{\Omega} \times\left[0, \tau_{\epsilon}\right]$, where $\sigma=\sigma(\epsilon)$. On $\left[0, \tau_{\epsilon}\right)$, recalling 2.13 we know that $0<u_{\epsilon}(x, t)<\tilde{A}$ for some $\tilde{A}$ which is independent of $\epsilon$. Multiplying the equation in $\left(\mathbb{P}_{\epsilon}\right)$ by $G_{\epsilon}^{\prime}\left(u_{\epsilon}\right)$ [39, 7], where

$$
G_{\epsilon}(s)=-\int_{s}^{\tilde{A}} g_{\epsilon}(r) \mathrm{d} r, \quad g_{\epsilon}(s)=-\int_{s}^{\tilde{A}} \frac{d r}{f_{\epsilon}(r)},
$$

and integrating, gives

$$
\int_{\Omega} G_{\epsilon}\left(u_{\epsilon}(x, T)\right) \mathrm{d} x+\int_{Q_{T}}\left[u_{\epsilon x x}^{2}-\left(v u_{\epsilon}^{m-n}-A u_{\epsilon}^{M-n}\right) u_{\epsilon x}^{2}\right] \mathrm{d} x \mathrm{~d} t=\int_{\Omega} G_{\epsilon}\left(u_{0 \epsilon}\right) \mathrm{d} x .
$$

If $m-n \neq-1$, then integrating the term $-\int_{Q_{T}} \nu u_{\epsilon}^{m-n} u_{\epsilon x}^{2}$ by parts yields

$$
\begin{aligned}
& \int_{\Omega} G_{\epsilon}\left(u_{\epsilon}(x, T)\right) \mathrm{d} x+\int_{Q_{T}} u_{\epsilon x x}^{2} \mathrm{~d} x \mathrm{~d} t+A \int_{Q_{T}} u_{\epsilon}^{M-n} u_{\epsilon x}^{2} \mathrm{~d} x \mathrm{~d} t \\
& \quad \leqslant \frac{1}{2} \int_{Q_{T}} u_{\epsilon x x}^{2} \mathrm{~d} x \mathrm{~d} t+\frac{1}{2(m-n+1)^{2}} \int_{Q_{T}} u_{\epsilon}^{2 m-2 n+2} \mathrm{~d} x \mathrm{~d} t+\int_{\Omega} G_{\epsilon}\left(u_{0 \epsilon}\right) \mathrm{d} x
\end{aligned}
$$

and recalling 2.11 we obtain

$$
\int_{\Omega} G_{\epsilon}\left(u_{\epsilon}(x, T)\right) \mathrm{d} x+\int_{Q_{T}} \frac{1}{2} u_{\epsilon x x}^{2} \mathrm{~d} x \mathrm{~d} t \leqslant c_{12} \int_{Q_{T}} u_{\epsilon}^{-2} \mathrm{~d} x \mathrm{~d} t+\int_{\Omega} G_{\epsilon}\left(u_{0 \epsilon}\right) \mathrm{d} x .
$$

If $m-n=-1$, the term $\left(\ln u_{\epsilon}\right)^{2}$ replaces $u_{\epsilon}^{2 m-2 n+2} /(m-n+1)^{2}$ in 2.15). Then noting that

$$
\ln ^{2}(s) \leqslant c_{13} s^{-2}, \quad 0<s<\tilde{A}<\infty,
$$

the estimate (2.16) again follows.

Since by 2.4$)$,

$$
\epsilon s^{-4} \leqslant \frac{1}{f_{\epsilon}(s)}, \quad s>0
$$

it now follows easily that

$$
\int_{\Omega} G_{\epsilon}\left(u_{\epsilon}(x, T)\right) \mathrm{d} x+\int_{Q_{T}} \frac{1}{2} u_{\epsilon x x}^{2} \mathrm{~d} x \mathrm{~d} t \leqslant \frac{c_{14}}{\epsilon} \int_{Q_{T}} G_{\epsilon}\left(u_{\epsilon}\right) \mathrm{d} x \mathrm{~d} t+c_{15} T+\int_{\Omega} G_{\epsilon}\left(u_{0 \epsilon}\right) \mathrm{d} x .
$$

Using 2.3, 2.17), we find that $\int_{\Omega} G_{\epsilon}\left(u_{0 \epsilon}\right) \mathrm{d} x \leqslant c_{16}$. Now we may use Gronwall's inequality to conclude that

$$
\int_{\Omega} G_{\epsilon}\left(u_{\epsilon}(x, T)\right) \mathrm{d} x \leqslant D_{\epsilon}(T)<\infty, \quad T \in\left[0, \tau_{\epsilon}\right),
$$

where for all $0<\epsilon \ll 1, D_{\epsilon}(T)$ is a positive increasing function of $T$ defined on [0, $\left.\infty\right)$. As in [7], 2.18 can be seen to imply positivity.

The solution, $u_{\epsilon}(x, t)$, may now be extended to exist globally, as in [7, 24]. Select $\tilde{f}_{\epsilon} \in \mathcal{C}^{2}(\mathbb{R})$ such that $\tilde{f}_{\epsilon}(s) \equiv f_{\epsilon}(s)$ for $s \geqslant 2 \sigma$, and $\tilde{f}_{\epsilon}(s) \geqslant f_{\epsilon}(\sigma)$ for all $s \in \mathbb{R}$. Thus $u_{\epsilon}$ also constitutes a weak solution of

$$
u_{\epsilon t}+\left\{\tilde{f}_{\epsilon}\left(u_{\epsilon}\right)\left(u_{\epsilon x x x}+h^{\prime}\left(u_{\epsilon}\right) u_{\epsilon x}\right)\right\}_{x}=0,
$$


satisfying the same initial and boundary conditions as before. Set

$$
v_{\epsilon}(x, t)=\int_{-a}^{x}\left(u_{\epsilon}(\xi, t)-\overline{u_{\epsilon}}(t)\right) \mathrm{d} \xi .
$$

The regularity and positivity of $u_{\epsilon}(x, t)$ imply that $v_{\epsilon}(x, t)$ is well defined in $D=\bar{\Omega} \times\left(0, \tau_{\epsilon}\right)$ and satisfies

$$
\left\{\begin{array}{l}
v_{\epsilon t}+\tilde{f}_{\epsilon}\left(u_{\epsilon}(x, t)\right)\left\{v_{\epsilon x x x x}+v u_{\epsilon}^{m-n} v_{\epsilon x x}-A u_{\epsilon}^{M-n} v_{\epsilon x x}\right\}=0 \\
v_{\epsilon}( \pm a, t)=v_{\epsilon x x}( \pm a, t)=0 .
\end{array}\right.
$$

Using parabolic regularity results for $v_{\epsilon}$, enhanced regularity may be obtained for $u_{\epsilon}$ and hence for the $u_{\epsilon}$-dependent coefficients in (2.19). Returning again to (2.19), additional regularity is obtained for $v_{\epsilon}$, which allows us to conclude that $u_{\epsilon}\left(x, \tau_{\epsilon}\right) \in \mathcal{C}^{4, \lambda}(\Omega)$. Therefore the solution may be continued, in contradiction to the assumed maximality.

Having demonstrated the global existence of positive approximants, $u_{\epsilon}$, the existence of a sequence $\left\{u_{\epsilon_{k}}\right\}$ converging uniformly to a solution of $(\mathbb{P})$ on $\overline{Q_{T}}$, for all $0<T<\infty$, as $\epsilon_{k} \rightarrow 0$, is now implied by Arzelà-Ascoli and the uniform Hölder estimates, [2.13), as in [7].

REMARK 2.3 It can be readily verified that the results of Theorem 1 remain valid when $m-n=$ -2 and $v=-1$, if the initial conditions satisfy the additional constraint that $-\int_{\Omega} H\left(u_{0}\right) \mathrm{d} x<\infty$.

REMARK 2.4 The existence of weak solutions for 1.3 and for 1.4 (a), with $h^{\prime}(u)$ replacing $v u^{m-n}-A u^{M-n}$ in Definition 1, can be concluded for initial data satisfying 2.1, 2.3 , by verifying that (2.8) holds for (1.3), that (2.9) holds for (1.4) (a), and that 2.16) holds for both (1.3) and (1.4)(a) and then arguing as above.

\section{Strong entropy-energy solutions}

To get strong entropy-energy solutions, we derive local entropy estimates [2], which give us strong solutions [2, 12], then derive a local energy estimate [25]. In obtaining the local entropy estimates and strong solutions, we follow [2] closely. Throughout this section the parameters will be assumed to satisfy the conditions in Theorem 1. Moreover, when referring to solutions of $(\mathbb{P})$ and $\left(\mathbb{P}_{\epsilon}\right)$, we shall assume that $u_{0}$ satisfies 2.1 and $u_{0 \epsilon}$ satisfies 2.3. Some further restrictions will be introduced later.

Before deriving the entropy estimates, we present a lemma, which constitutes a refinement of Theorem 3.1 in [2], and which is useful when deducing regularity results from entropy estimates.

LEMma 3.1 Let $u(x, t)$ be a weak solution of $(\mathbb{P})$ obtained as the limit of a subsequence of solutions $u_{\epsilon}(x, t)$ of $\left(\mathbb{P}_{\epsilon}\right)$. Suppose that for some $\alpha \in(1 / 2-n, 2-n)$, there exist constants $c_{1}, c_{2}$, and $\delta>0$, which do not depend on $\epsilon$, such that

$$
\int_{Q_{T}} u_{\epsilon}^{\alpha+n-2 \gamma+1}\left(u_{\epsilon}^{\gamma}\right)_{x x}^{2} \mathrm{~d} x \mathrm{~d} t \leqslant c_{1}
$$

and

for all $\gamma$ satisfying

$$
\int_{Q_{T}} u_{\epsilon}^{\alpha+n-3} u_{\epsilon_{x}}^{4} \mathrm{~d} x \mathrm{~d} t \leqslant c_{2}
$$

$$
\frac{1+n+\alpha}{3} \leqslant \gamma \leqslant \frac{1+n+\alpha}{3}+\delta
$$

then $u^{1 / \beta}(\cdot, t) \in \mathcal{C}^{1}([-a, a])$ for all $\beta \in(0,3 /(n+\alpha+1))$, for almost every $t>0$. 
Proof. For any $0<\beta<3 /(n+\alpha+1)$, we may choose $\gamma$ satisfying (3.3) such that $0<\beta \gamma<1$. Setting $q=4-(1+n+\alpha) / \gamma$ and arguing as in the proof of [2, Lemma 3.1], it follows from (3.1), (3.2) that for almost every $t>0$ there exists a $C_{1}(t)<\infty$ such that

if $u(y, t)=0$ for some $y \in[-a, a]$, then

$$
\left|\left(u^{\gamma}\right)_{x}\right|^{(4-q) / q}(x, t) \leqslant C_{1}(t)|x-y|^{(q-1) / q} \quad \text { for } x \in[-a, a] .
$$

From (3.4, we find by integrating that for almost every $t>0$, there exists a $C_{2}(t)<\infty$ such that

if $u(y, t)=0$ for some $y \in[-a, a]$, then

$$
u(x, t) \leqslant C_{2}(t)|x-y|^{3 /(\alpha+n+1)} \quad \text { for } x \in[-a, a] .
$$

Since $0<\beta \gamma<1$, we may combine 3.5 and 3.4 to see that for almost every $t>0$, there exists a $C_{3}(t)<\infty$ such that

if $u(y, t)=0$ for some $y \in[-a, a]$, then for $x \in[-a, a]$,

$$
\left|\left(u^{1 / \beta}\right)_{x}(x, t)\right| \leqslant C_{3}(t)|x-y|^{\frac{3}{4-q}}|x-y|^{\frac{q-1}{4-q}} \leqslant C_{3}(t)|x-y|^{\mu},
$$

where $\mu=\frac{1}{\beta} \frac{3}{\alpha+n+1}-1>0$ and $C_{3}(t)<\infty$.

From Lemma 3.1, two simple but useful corollaries follow.

Corollary $3.2([2])$ Let $u(x, t)$ be a weak solution of $(\mathbb{P})$ obtained as the limit of a subsequence of solutions $u_{\epsilon}(x, t)$ of $\left(\mathbb{P}_{\epsilon}\right)$. Suppose that for some $\alpha \in(1 / 2-n, 2-n)$, there exist constants $c_{1}$, $c_{2}$, and $\delta>0$, which do not depend on $\epsilon$, such that for all $\gamma$ satisfying (3.3), the estimates (3.1) and 3.2 hold. Then $u(\cdot, t) \in \mathcal{C}^{1}([-a, a])$ for almost every $t>0$.

Proof. If $\alpha \in(1 / 2-n, 2-n)$, then $3 /(\alpha+n+1) \in(1,2)$. Hence $1 \in(0,3 /(n+\alpha+1))$.

REMARK 3.3 If $u(\cdot, t) \in \mathcal{C}^{1}([-a, a])$ for almost every $t>0$, then $u(x, t)$ is said to be a strong solution in the sense of Bernis [4].

COROLlary 3.4 Let $u(x, t)$ be a weak solution of $(\mathbb{P})$ obtained as the limit of a subsequence of solutions $u_{\epsilon}(x, t)$ of $\left(\mathbb{P}_{\epsilon}\right)$. Let $\Psi$ denote a nonempty subset of $(1 / 2-n, 2-n)$. If for all $\alpha \in \Psi$, there exist constants $c_{1}, c_{2}$, and $\delta>0$, which do not depend on $\epsilon$, such that for all $\gamma$ satisfying [3.3, the estimates 3.1 and 3.2 hold, then $u^{1 / \beta}(\cdot, t) \in \mathcal{C}^{1}([-a, a])$ for all $\beta \in(0,3 /(n+\inf \Psi+1))$ for almost every $t>0$.

Proof. The result is an immediate consequence of Lemma 3.1 .

We now derive our primary entropy estimates. Let

$$
\zeta \in \mathcal{C}^{4}([-a, a]) \quad \text { with support in }(-a, a) \text { and } \zeta \geqslant 0,
$$

or $\zeta \equiv 1$, and let $[2,12$

$$
G_{\epsilon}(s)=\frac{\epsilon s^{\alpha+n-3}}{(\alpha+n-4)(\alpha+n-3)}+\frac{s^{\alpha+1}}{\alpha(\alpha+1)},
$$


where $\alpha \in(1 / 2-n, 2-n) \backslash\{0,-1\}$. Using $\zeta^{4} G_{\epsilon}^{\prime}\left(u_{\epsilon}\right)$ to test $\left(\mathbb{P}_{\epsilon}\right)$ on $Q_{T}=\Omega \times(0, T), 0<T<\infty$, and treating the terms which also appear in the classical thin film equation as they were treated in [2], we conclude that for any $\gamma$ satisfying

$$
\frac{t+1-\sqrt{(t-2)(1-2 t)}}{3}<\gamma<\frac{t+1+\sqrt{(t-2)(1-2 t)}}{3},
$$

where $t=\alpha+n$, there exist positive constants, $c_{3}, c_{4}$, which do not depend on $\epsilon$, such that

$$
\begin{aligned}
\int_{\Omega} \zeta^{4} G_{\epsilon}\left(u_{\epsilon}(x, T)\right) \mathrm{d} x & +c_{3}\left[\int_{Q_{T}} \zeta^{4} u_{\epsilon}^{\alpha+n-2 \gamma+1}\left(u_{\epsilon}^{\gamma}\right)_{x x}^{2} \mathrm{~d} x \mathrm{~d} t+\int_{Q_{T}} \zeta^{4} u_{\epsilon}^{\alpha+n-3} u_{\epsilon x}^{4} \mathrm{~d} x \mathrm{~d} t\right] \\
& \leqslant \int_{\Omega} \zeta^{4} G_{\epsilon}\left(u_{0 \epsilon}\right) \mathrm{d} x+c_{4} \int_{Q_{T}}\left(\left|\zeta_{x}\right|^{4}+\left|\zeta \zeta_{x x}\right|^{2}\right) u_{\epsilon}^{n+\alpha+1} \mathrm{~d} x \mathrm{~d} t+I,
\end{aligned}
$$

where

$$
I:=-\int_{Q_{T}} \zeta^{4} g_{\epsilon}\left(u_{\epsilon}\right)\left\{f_{\epsilon}\left(u_{\epsilon}\right)\left(v u_{\epsilon}^{m-n} u_{\epsilon x}-A u_{\epsilon}^{M-n} u_{\epsilon x}\right)\right\}_{x} \mathrm{~d} x \mathrm{~d} t, \quad g_{\epsilon}\left(u_{\epsilon}\right):=G_{\epsilon}^{\prime}\left(u_{\epsilon}\right) .
$$

Integrating $I$ by parts yields

$$
\begin{aligned}
I= & \int_{Q_{T}} \zeta^{4} g_{\epsilon}^{\prime}\left(u_{\epsilon}\right) f_{\epsilon}\left(u_{\epsilon}\right)\left(v u_{\epsilon}^{m-n} u_{\epsilon x}^{2}-A u_{\epsilon}^{M-n} u_{\epsilon x}^{2}\right) \mathrm{d} x \mathrm{~d} t \\
& +\int_{Q_{T}} 4 \zeta^{3} \zeta_{x} g_{\epsilon}\left(u_{\epsilon}\right) f_{\epsilon}\left(u_{\epsilon}\right)\left(v u_{\epsilon}^{m-n} u_{\epsilon x}-A u_{\epsilon}^{M-n} u_{\epsilon x}\right) \mathrm{d} x \mathrm{~d} t=: I_{a}+I_{b}
\end{aligned}
$$

The term $I_{a}$ may be written as

$$
I_{a}=\int_{Q_{T}} \zeta^{4}\left(v u_{\epsilon}^{\alpha+m-1}-A u_{\epsilon}^{\alpha+M-1}\right) u_{\epsilon x}^{2} \mathrm{~d} x \mathrm{~d} t .
$$

For $v=-1$, both terms in 3.10 are nonpositive. For $v=+1$, we estimate

$$
\begin{aligned}
I_{a} \leqslant & \delta \int_{Q_{T}} \zeta^{4} u_{\epsilon}^{\alpha+n-3} u_{\epsilon x}^{4} \mathrm{~d} x \mathrm{~d} t+c_{5}(\delta) \int_{Q_{T}} \zeta^{4} u_{\epsilon}^{\alpha+2 m-n+1} \mathrm{~d} x \mathrm{~d} t \\
& -A \int_{Q_{T}} \zeta^{4} u_{\epsilon}^{\alpha+M-1} u_{\epsilon x}^{2} \mathrm{~d} x \mathrm{~d} t,
\end{aligned}
$$

where $\delta>0$ is arbitrary.

With regard to $I_{b}$, integration by parts gives

$$
I_{b}=-\int_{Q_{T}} 4\left(\zeta^{3} \zeta_{x}\right)_{x}\left[\int_{0}^{u_{\epsilon}} g_{\epsilon}(s) f_{\epsilon}(s)\left[v s^{m-n}-A s^{M-n}\right] \mathrm{d} s\right] \mathrm{d} x \mathrm{~d} t
$$

As noted in [2], $\left|g_{\epsilon}\left(u_{\epsilon}\right) f_{\epsilon}\left(u_{\epsilon}\right)\right| \leqslant c_{6} u_{\epsilon}^{n+\alpha}$. Thus, recalling 2.11] and that $M>m$,

$$
I_{b} \leqslant c_{7} \int_{Q_{T}}\left|\left(\zeta^{3} \zeta_{x}\right)_{x}\right| u_{\epsilon}^{\alpha+m+1} \mathrm{~d} x \mathrm{~d} t
$$


If $v=-1$, we may combine the estimates on $I_{a}$ and $I_{b}$ to obtain

$$
\begin{aligned}
& \int_{\Omega} \zeta^{4} G_{\epsilon}\left(u_{\epsilon}(x, T)\right) \mathrm{d} x+A \int_{Q_{T}} \zeta^{4} u_{\epsilon}^{\alpha+M-1} u_{\epsilon x}^{2} \mathrm{~d} x \mathrm{~d} t \\
& +c_{3}\left[\int_{Q_{T}} \zeta^{4} u_{\epsilon}^{\alpha+n-2 \gamma+1}\left(u_{\epsilon}^{\gamma}\right)_{x x}^{2} \mathrm{~d} x \mathrm{~d} t+\int_{Q_{T}} \zeta^{4} u_{\epsilon}^{\alpha+n-3} u_{\epsilon x}^{4} \mathrm{~d} x \mathrm{~d} t\right] \\
& \quad+\int_{Q_{T}} \zeta^{4} u_{\epsilon}^{\alpha+m-1} u_{\epsilon x}^{2} \mathrm{~d} x \mathrm{~d} t \leqslant \int_{\Omega} \zeta^{4} G_{\epsilon}\left(u_{0 \epsilon}\right) \mathrm{d} x+I I,
\end{aligned}
$$

where

$$
I I=c_{4} \int_{Q_{T}}\left(\left|\zeta_{x}\right|^{4}+\left|\zeta \zeta_{x x}\right|^{2}\right) u_{\epsilon}^{n+\alpha+1} \mathrm{~d} x \mathrm{~d} t+c_{7} \int_{Q_{T}}\left|\left(\zeta^{3} \zeta_{x}\right)_{x}\right| u_{\epsilon}^{\alpha+m+1} \mathrm{~d} x \mathrm{~d} t .
$$

Similarly, if $v=+1$, the estimates yield

$$
\begin{aligned}
& \int_{\Omega} \zeta^{4} G_{\epsilon}\left(u_{\epsilon}(x, T)\right) \mathrm{d} x+A \int_{Q_{T}} \zeta^{4} u_{\epsilon}^{\alpha+M-1} u_{\epsilon x}^{2} \mathrm{~d} x \mathrm{~d} t \\
& +c_{8}\left[\int_{Q_{T}} \zeta^{4} u_{\epsilon}^{\alpha+n-2 \gamma+1}\left(u_{\epsilon}^{\gamma}\right)_{x x}^{2} \mathrm{~d} x \mathrm{~d} t+\int_{Q_{T}} \zeta^{4} u_{\epsilon}^{\alpha+n-3} u_{\epsilon x}^{4} \mathrm{~d} x \mathrm{~d} t\right] \\
& \quad \leqslant c_{5} \int_{Q_{T}} \zeta^{4} u_{\epsilon}^{\alpha+2 m-n+1} \mathrm{~d} x \mathrm{~d} t+\int_{\Omega} \zeta^{4} G_{\epsilon}\left(u_{0 \epsilon}\right) \mathrm{d} x+I I .
\end{aligned}
$$

To obtain bounds from 3.13, 3.14, we impose certain conditions on $\alpha$ and on the initial data.

REMARK 3.5 Suppose that $u_{0}$ satisfies (2.1) and $0<n<3$. Defining

$$
\alpha^{*}= \begin{cases}1 / 2-n, & 0<n \leqslant 3 / 2 \\ -1, & 3 / 2<n<3\end{cases}
$$

we see that $\alpha^{*}+1 \geqslant 0, \alpha^{*} \in[1 / 2-n, 2-n)$, and $\int_{\Omega} \zeta^{4} u_{0}^{\alpha+1} \mathrm{~d} x<\infty$ for all $\alpha \in\left(\alpha^{*}, 2-n\right)$ and $\zeta \in \mathcal{C}^{4}([-a, a])$.

In view of the above remark, we introduce

Definition 2 Suppose that $u_{0}$ satisfies 2.1 and $\zeta \in \mathcal{C}^{4}([-a, a])$. Then we define $\alpha_{0}(\zeta)$ to be the infimum among all $\alpha$ such that $\alpha>1 / 2-n$ and

$$
\begin{array}{ll}
\int_{\Omega} \zeta^{4} u_{0}^{\alpha+1} \mathrm{~d} x<\infty & \text { if } \alpha \neq-1 \\
\int_{\Omega} \zeta^{4}\left|\ln u_{0}\right| \mathrm{d} x<\infty & \text { if } \alpha=-1 .
\end{array}
$$

Remark 3.5 and the definition of $\alpha_{0}(\zeta)$ imply that if $0<n<3$, then

$$
1 / 2-n \leqslant \alpha_{0}(\zeta) \leqslant \alpha^{*}<2-n .
$$

With regard to the stable case, the theorem below follows essentially as in [2, 20]. 
Theorem 2 (The stable case) Suppose that $v=-1, m-n \geqslant-2$, with $m<M$ if $A>0$, and with $-\int_{\Omega} H\left(u_{0}\right) \mathrm{d} x<\infty$ if $m-n=-2$.

(i) Let $\beta \in\left(0, \beta_{0}\right)$ where $\beta_{0}=\max \left\{\frac{3}{n+\alpha_{0}+1}, \frac{1}{m+\alpha_{0}+1}\right\}$ and $\alpha_{0}=\alpha_{0}(\zeta=1)$. Then $u^{1 / \beta}(\cdot, t) \in$ $\mathcal{C}^{1}([-a, a])$ for almost every $t>0$.

(ii) Let $\zeta$ satisfy (3.7). Then, for any $\alpha \in\left(\max \left\{\alpha_{0}(\zeta),-m-1\right\}, 2-n\right) \backslash\{0,-1\}$ and for any $\gamma$ satisfying 3.8 ,

$$
\begin{gathered}
\frac{1}{\alpha(\alpha+1)} \int_{\Omega} \zeta^{4} u^{1+\alpha}(x, T) \mathrm{d} x+c_{1}\left[\int_{P} \zeta^{4} u^{\alpha+n-2 \gamma+1}\left(u^{\gamma}\right)_{x x}^{2} \mathrm{~d} x \mathrm{~d} t+\int_{Q_{T}} \zeta^{4} u^{\alpha+n-3} u_{x}^{4} \mathrm{~d} x \mathrm{~d} t\right] \\
+\left[\int_{Q_{T}} \zeta^{4} u^{\alpha+m-1} u_{x}^{2} \mathrm{~d} x \mathrm{~d} t+A \int_{Q_{T}} \zeta_{x}^{4} u^{\alpha+M-1} u_{x}^{2} \mathrm{~d} x \mathrm{~d} t\right] \\
\leqslant c_{2} \int_{Q_{T}}\left(\left|\zeta_{x}\right|^{4}+\left|\zeta \zeta_{x x}\right|^{2}\right) u^{n+\alpha+1} \mathrm{~d} x \mathrm{~d} t+c_{3} \int_{Q_{T}}\left|\left(\zeta^{3} \zeta_{x}\right)_{x}\right| u^{\alpha+m+1} \mathrm{~d} x \mathrm{~d} t \\
+\frac{1}{\alpha(\alpha+1)} \int_{\Omega} \zeta^{4} u_{0}^{\alpha+1} \mathrm{~d} x .
\end{gathered}
$$

Proof. Part (i) follows by setting $\zeta=1$ in (3.13), invoking Lemma 3.1, and noting the Hölder regularity implied by the boundedness of $\int_{Q_{T}}\left(u^{(\alpha+m+1) / 2}\right)_{x}^{2} \mathrm{~d} x \mathrm{~d} t$. Part (ii) follows easily from (3.13) by letting $\epsilon \rightarrow 0$ and noting that $\alpha+m+1$ and $\alpha+n+1$ are positive in the indicated parameter range.

Remark 3.6 If $m<0$ and $m-n<-3 / 2$, then the Infinite Speed of Propagation Property holds for the solutions discussed in Theorem 2 due to the boundedness of $\int_{Q_{T}}\left(u^{(\alpha+m+1) / 2}\right)_{x}^{2} \mathrm{~d} x \mathrm{~d} t$. See [20, Corollary 2.1].

Theorem 3 (The unstable case) Let $v=1, A \geqslant 0, n>0, m-n>-3 / 2, m-n<2$ if $A=0$, and $m<M$ if $A>0$.

(i) Let $\alpha_{0}=\alpha_{0}(\zeta=1), \alpha_{1}=\max \left\{\alpha_{0},-2 m+n-1\right\}$, and $\beta_{0}=3 /\left(n+\alpha_{1}+1\right)$. Then $u^{1 / \beta}(\cdot, t) \in$ $\mathcal{C}^{1}([-a, a])$ for all $\beta \in\left(0, \beta_{0}\right)$, for almost every $t>0$.

(ii) For any $\zeta$ satisfying 3.7], let $\alpha_{2}=\max \left\{\alpha_{0}(\zeta),-2 m+n-1,-m-1\right\}$. Then, for any $\alpha \in$ $\left(\alpha_{2}, 2-n\right) \backslash\{0,-1\}$ and for any $\gamma$ satisfying 3.8 ,

$$
\begin{aligned}
\frac{1}{\alpha(\alpha+1)} \int_{\Omega} \zeta^{4} u^{1+\alpha}(x, T) \mathrm{d} x & +A \int_{Q_{T}} \zeta^{4} u^{\alpha+M-1} u_{x}^{2} \mathrm{~d} x \mathrm{~d} t \\
& +c_{1}\left[\int_{P} \zeta^{4} u^{\alpha+n-2 \gamma+1}\left(u^{\gamma}\right)_{x x}^{2} \mathrm{~d} x \mathrm{~d} t+\int_{Q_{T}} \zeta^{4} u^{\alpha+n-3} u_{x}^{4} \mathrm{~d} x \mathrm{~d} t\right] \\
\leqslant & c_{2} \int_{Q_{T}}\left(\left|\zeta_{x}\right|^{4}+\left|\zeta \zeta_{x x}\right|^{2}\right) u^{n+\alpha+1} \mathrm{~d} x \mathrm{~d} t+c_{3} \int_{Q_{T}}\left|\left(\zeta^{3} \zeta_{x}\right)_{x}\right| u^{\alpha+m+1} \mathrm{~d} x \mathrm{~d} t \\
& +c_{4} \int_{Q_{T}} \zeta^{4} u^{\alpha+2 m-n+1} \mathrm{~d} x \mathrm{~d} t+\frac{1}{\alpha(\alpha+1)} \int_{\Omega} \zeta^{4} u_{0}^{\alpha+1} \mathrm{~d} x
\end{aligned}
$$

Proof. To prove part (i), note that the condition $m-n>-3 / 2$ implies that $\alpha_{1} \in[1 / 2-n, 2-n$ ), and that $\alpha_{1} \geqslant \alpha_{0}, \alpha_{1}+2 m-n+1 \geqslant 0$; then invoke Lemma 3.1 with $\zeta=1$. Part (ii) follows by noting that $\alpha_{2} \in[1 / 2-n, 2-n), \alpha>\alpha_{0}, \alpha+m+1>0$, and $\alpha+2 m-n+1 \geqslant 0$, and then letting $\epsilon \rightarrow 0$ in (3.14). 
REMARK 3.7 The results given in Theorem 3 also hold for the exceptional cases (1.3), 1.4)(a), with $A=0$ and with $m-n$ assuming the values -1 and $m-n=0$, respectively. This can be easily demonstrated by following the arguments above, once one notices that estimates (3.11), (3.12) also hold for 11.3 , 1.4. (a) when the value of $m-n$ is -1 or 0 , respectively, by utilizing the bounds (1.8), (1.9).

REMARK 3.8 When $0<n<3$, Corollary 3.2 and 3.16 imply that under the assumptions in Theorems 2 and 3 , the weak solutions obtained as the limit of a subsequence of $\left\{u_{\epsilon}(x, t)\right\}$, the solutions to $\left(\mathbb{P}_{\epsilon}\right)$, are in fact strong solutions in the sense of Bernis [4]. In particular this implies that solutions with compact support have zero contact angle at the edge of the support.

Using (3.15)-3.16, one can check that in the context of Theorems 2 and 3 ,

$$
\beta_{0} \geqslant \begin{cases}2, & 0<n \leqslant 3 / 2, \\ 3 / n, & 3 / 2<n<3 .\end{cases}
$$

These bounds correspond to the bounds which were obtained in [2] for the thin film equation [1.1]. The regularity indicated in (3.19) also corresponds to the contact line regularity of the self-similar source type solutions which were demonstrated in [1] to exist for (1.6) when $0<n<3, v= \pm 1$, $A=0$, and $m=n+2$. In this sense the regularity obtained is sharp, though additional regularity is provided by the terms $\int_{Q_{T}}\left(u^{(\alpha+m+1) / 2}\right)_{x}^{2} \mathrm{~d} x, \int_{Q_{T}} A\left(u^{(\alpha+M+1) / 2}\right)_{x}^{2} \mathrm{~d} x$, for some parameter values.

REMARK 3.9 For $0<n<3$, with regard to the terms in (2.2) which do not depend on $m$ or $M$, since the regularity obtained is the same as the regularity which was obtained for [1.1) in [2], these terms can be interpreted in the same sense as in [12], which is distributional for $3 / 8<n<3$ and is in the weaker sense of 2.2 for $0<n \leqslant 3 / 8$. With regard to the terms in 2.2 which depend on $m$ and $M$, these terms may be readily seen to be interpretable in a suitable distributional sense if $m>-1$ and in the weaker sense of (2.2) for $m \leqslant-1$. The details of the case $v=-1,0<m<1$ are discussed in [11].

In the case of strong slippage, in which $0<n<2$, the local entropy estimates provided by Theorems 2$]$ and 3 can be used to prove the finite speed propagation property for the strong solutions obtained there when $m>n / 2$ (see $\S 4$ ). In the case of weak slippage in which

$$
2 \leqslant n<3,
$$

the finite speed propagation property for the standard thin film equation (1.1) and $u_{0} \in H^{1}(\Omega)$ was proved by F. Bernis [5] using local energy estimates, which were derived there. The proof of the energy estimates is based on certain integral inequalities for smooth functions which are positive on some interval (see [6]). Our proof of the finite speed of propagation property for (1.6) for $m>n / 2$ in the case of weak slippage (3.20) is based on combined use of local entropy estimates, which were derived in Theorems 2 and 3 , and local energy estimates which are derived below in Theorem 4 . The derivation of the local energy estimates makes use of a generalization of the Bernis inequalities mentioned earlier, for nonnegative periodic functions (see Lemma 6.3). Somewhat similar methodologies were employed in [30, 25] where local energy estimates were also derived, and which also relied on combined use of local entropy and energy estimates. Notably, the combination of local energy and entropy estimates used here and in [25] relies on using local entropy estimates with $-1<\alpha<0$; previous approaches relied on local entropy estimates with $\alpha>0$ in proving the finite speed of propagation property [2, 12, 20]. 
TheOREM 4 Suppose that $v= \pm 1,2 \leqslant n<3, A \geqslant 0$, and that $m<M$ if $A>0$, and $m<n+2$ if $A=0$ and $v=1$. Suppose moreover that if $v=-1$, then $m-\frac{3}{4} n \geqslant-1$, and if $v=1$, then $m-\frac{2}{3} n>-2 / 3$ whenever $2 \leqslant n<5 / 2$, and $m-n>-3 / 2$ whenever $5 / 2 \leqslant n<3$. Under these assumptions, the strong solutions obtained in Theorems 2 and 3 satisfy the local energy estimate

$$
\begin{aligned}
\int_{\Omega} \zeta^{6}\left|u_{x}(x, T)\right|^{2} \mathrm{~d} x+d_{1} & \int_{Q_{T}} \zeta^{6}\left(\left(u^{(n+2) / 6}\right)_{x}^{6}+\left|\left(u^{(n+2) / 3}\right)_{x x}\right|^{3}+\left(u^{(n+2) / 2}\right)_{x x x}^{2}\right) \mathrm{d} x \mathrm{~d} t \\
& +d_{1} \int_{Q_{T} \cap\{u>0\}} \zeta^{6} u^{n} u_{x x x}^{2} \mathrm{~d} x \mathrm{~d} t \\
\leqslant & \int_{\Omega} \zeta^{6}\left|u_{0 x}(x)\right|^{2} \mathrm{~d} x+d_{2} \int_{Q_{T}} u^{n+2}\left(\left|\zeta_{x}\right|^{6}+\left|\zeta \zeta_{x x}\right|^{3}\right) \mathrm{d} x \mathrm{~d} t \\
& -\int_{Q_{T} \cap\{u>0\}}\left(v u^{m} u_{x}-A u^{M} u_{x}\right)\left(u_{x} \zeta^{6}\right)_{x x} \mathrm{~d} x \mathrm{~d} t
\end{aligned}
$$

where $\zeta(x)$ is an arbitrary nonnegative function from $\mathcal{C}^{4}([-a, a])$.

Proof. In order to derive the local energy estimate, we test the equation in the approximating problem, $\left(\mathbb{P}_{\epsilon}\right)$, with $-\left(\zeta^{6} u_{\epsilon x}\right)_{x}$, and easily deduce that

$$
\begin{aligned}
\int_{\Omega} \frac{\zeta^{6}}{2}\left|u_{\epsilon x}(x, T)\right|^{2} \mathrm{~d} x+\int_{Q_{T}} \zeta^{6} f_{\epsilon}\left(u_{\epsilon}\right)\left|u_{\epsilon x x x}\right|^{2} \mathrm{~d} x \mathrm{~d} t \\
=-\int_{Q_{T}} f_{\epsilon}\left(u_{\epsilon}\right) u_{\epsilon x x x}\left[2 u_{\epsilon x x}\left(\zeta^{6}\right)_{x}+u_{\epsilon x}\left(\zeta^{6}\right)_{x x}\right] \mathrm{d} x \mathrm{~d} t \\
\quad-\int_{Q_{T}} f_{\epsilon}\left(u_{\epsilon}\right)\left(v u_{\epsilon}^{m-n} u_{\epsilon x}-A u_{\epsilon}^{M-n} u_{\epsilon x}\right)\left(u_{\epsilon x} \zeta^{6}\right)_{x x} \mathrm{~d} x \mathrm{~d} t+\int_{\Omega} \frac{\zeta^{6}}{2}\left|u_{0 \epsilon x}\right|^{2} \mathrm{~d} x
\end{aligned}
$$

Assuming (3.20) and noting 3.15, 3.16), it is easy to check that $\max \left\{\alpha^{*},-m-1\right\}<0$ in Theorem 2 and that $\max \left\{\alpha^{*},-2 m+n-1,-m-1\right\}<0$ in Theorem 3 . Hence if $n$ satisfies 3.20, then for arbitrary $\zeta$ satisfying (3.7) or $\zeta=1, \alpha$ may be chosen to be fixed and to satisfy

$$
-1<\alpha<0
$$

in addition to satisfying the constraints indicated in part (ii) of either theorem.

For $\alpha$ satisfying 3.23, we have, due to 1.15,

$$
\int_{\Omega} u_{\epsilon}^{1+\alpha}(x, T) \mathrm{d} x \leqslant|\Omega|^{-\alpha}\left(\int_{\Omega} u_{\epsilon}(x, T) \mathrm{d} x\right)^{1+\alpha}=|\Omega|^{-\alpha}\left(\int_{\Omega} u_{0 \epsilon} \mathrm{d} x\right)^{1+\alpha} \leqslant d_{3} .
$$

Setting $\zeta=1$ in 3.13 and employing 2.3, 3.24, we obtain the following inequality when $v=-1$ :

$$
\begin{aligned}
c_{3}\left[\int_{Q_{T}} u_{\epsilon}^{\alpha+n-2 \gamma+1}\left(u_{\epsilon}^{\gamma}\right)_{x x}^{2} \mathrm{~d} x \mathrm{~d} t+\int_{Q_{T}} u_{\epsilon}^{\alpha+n-3} u_{\epsilon x}^{4} \mathrm{~d} x \mathrm{~d} t\right] & +\int_{Q_{T}}\left(u_{\epsilon}^{\alpha+m-1}+A u_{\epsilon}^{\alpha+M-1}\right) u_{\epsilon x}^{2} \mathrm{~d} x \mathrm{~d} t \\
& \leqslant \int_{\Omega} G_{\epsilon}\left(u_{0 \epsilon}\right) \mathrm{d} x+d_{4} \leqslant d_{5}
\end{aligned}
$$


Similarly, setting $\zeta=1$ in 3.14, employing 2.3, 3.24, and noting that the assumptions on $\alpha$ imply that $\alpha>\alpha_{2} \geqslant-2 m+n-1$, we obtain the following inequality when $v=1$ :

$$
\begin{aligned}
& c_{8}\left[\int_{Q_{T}} u_{\epsilon}^{\alpha+n-2 \gamma+1}\left(u_{\epsilon}^{\gamma}\right)_{x x}^{2} \mathrm{~d} x \mathrm{~d} t+\int_{Q_{T}} u_{\epsilon}^{\alpha+n-3} u_{\epsilon x}^{4} \mathrm{~d} x \mathrm{~d} t\right] \\
& +\int_{Q_{T}} A u_{\epsilon}^{\alpha+M-1} u_{\epsilon x}^{2} \mathrm{~d} x \mathrm{~d} t \leqslant \int_{\Omega} G_{\epsilon}\left(u_{0 \epsilon}\right) \mathrm{d} x+c_{5} \int_{Q_{T}} u_{\epsilon}^{\alpha+2 m-n+1} \mathrm{~d} x \mathrm{~d} t+d_{6} \leqslant d_{7} .
\end{aligned}
$$

Next we pass to the limit $\epsilon \rightarrow 0$ in 3.22. First, setting $\zeta=1$ in 3.22 shows that for any $\delta>0$

$$
\begin{aligned}
& 2^{-1} \int_{\Omega}\left|u_{\epsilon x}(x, T)\right|^{2} \mathrm{~d} x+\int_{Q_{T}} f_{\epsilon}\left(u_{\epsilon}\right)\left|u_{\epsilon x x x}\right|^{2} \mathrm{~d} x \mathrm{~d} t \\
& \quad \leqslant 2^{-1} \int_{\Omega}\left|u_{0 \epsilon x}\right|^{2} \mathrm{~d} x+\left(1+A\left|\sup u_{\epsilon}\right|^{M-m}\right) \int_{Q_{T}} f_{\epsilon}\left(u_{\epsilon}\right) u_{\epsilon}^{m-n}\left|u_{\epsilon x}\right|\left|u_{\epsilon x x x}\right| \mathrm{d} x \mathrm{~d} t \\
& \quad \leqslant 2^{-1} \int_{\Omega}\left|u_{0 \epsilon x}\right|^{2} \mathrm{~d} x+\delta \int_{Q_{T}} f_{\epsilon}\left(u_{\epsilon}\right)\left|u_{\epsilon x x x}\right|^{2} \mathrm{~d} x \mathrm{~d} t+\int_{Q_{T}} u_{\epsilon}^{\alpha+n-3} u_{\epsilon x}^{4} \mathrm{~d} x \mathrm{~d} t \\
& \quad+c(\delta) \int_{Q_{T}} u_{\epsilon}^{4 m-2 n-(\alpha+n)+3} \mathrm{~d} x \mathrm{~d} t
\end{aligned}
$$

Suppose that

$$
4 m-2 n-(\alpha+n)+3 \geqslant 0
$$

then setting $\delta=1 / 2$ in 3.27 and using the estimates 3.25, 3.26, we deduce that

$$
\int_{\Omega}\left|u_{\epsilon x}(x, T)\right|^{2} \mathrm{~d} x+\int_{Q_{T}} f_{\epsilon}\left(u_{\epsilon}\right)\left|u_{\epsilon x x x}\right|^{2} \mathrm{~d} x \mathrm{~d} t<d_{8} .
$$

REMARK 3.10 In the context of Theorem 2, when $n$ satisfies 3.20 , we have $m>n-2 \geqslant 0$, hence $\max \left\{\alpha^{*},-m-1\right\}=\alpha^{*}=-1$. Thus for arbitrary initial data satisfying $[2.1]$ and $[2.3)$, the condition 3.28 is satisfied for some admissible $\alpha$ if

$$
m-\frac{3}{4} n \geqslant-1
$$

which is stronger than the previous constraint, $m-n>-2$. In the context of Theorem 3, when $n$ satisfies 3.20 , we have $m>n-2 \geqslant 0$; hence $\max \left\{\alpha^{*},-m-1\right\}=\alpha^{*}=-1$, but $\alpha$ there must also satisfy $\alpha>-2 m+n-1$. It is easy to check that 3.28 holds for some admissible $\alpha$ if and only if $m, n$ satisfy the condition

$$
3 m-2 n>-2 .
$$

Recalling the constraint $m-n>-3 / 2$ in Theorem 3, it is easy to check that 3.30) constitutes an additional constraint if $n<5 / 2$.

Using the estimates $3.29,3.25,3.26$, it is easy to check that the integrals on the right-hand side of (3.22) are uniformly bounded with respect to $\epsilon$ if 3.28 is satisfied. For $t>0, \eta>0$, $u_{\epsilon} \rightarrow u$ strongly in the space $\mathcal{C}^{4, \lambda}(\{u>\eta\})$. Therefore passage to the limit $\epsilon \rightarrow 0$ in all of the 
integrals in (3.22) over the domain $Q_{T} \cap\{u>\eta\}$ is straightforward. As to integrals over the domain $\{u<\eta\}$, we have, for example, by virtue of 3.28 ,

$$
\begin{aligned}
& \left|\int_{Q_{T} \cap\{u<\eta\}} f_{\epsilon}\left(u_{\epsilon}\right) u_{\epsilon x x x} u_{\epsilon x} u_{\epsilon}^{m-n} \zeta^{6} \mathrm{~d} x \mathrm{~d} t\right| \\
& \quad \leqslant c\left(\int_{Q_{T} \cap\{u<\eta\}} f_{\epsilon}\left(u_{\epsilon}\right)\left|u_{\epsilon x x x}\right|^{2} \mathrm{~d} x \mathrm{~d} t\right)^{1 / 2}\left(\int_{Q_{T} \cap\{u<\eta\}} u_{\epsilon}^{\alpha+n-3} u_{\epsilon x}^{4} \mathrm{~d} x \mathrm{~d} t\right)^{1 / 4} \\
& \quad \times\left(\int_{Q_{T} \cap\{u<\eta\}} u^{4 m-2 n-(\alpha+n)+3} \mathrm{~d} x \mathrm{~d} t\right)^{1 / 4} \leqslant c \eta^{(4 m-2 n-(\alpha+n)+3) / 4} \rightarrow 0 \quad \text { as } \eta \rightarrow 0 .
\end{aligned}
$$

Analogously, it is easy to check that all of the other integrals over $\{u<\eta\}$ on the right hand side of (3.22) are bounded from above by some continuous function, $h(\eta)$, such that $h(\eta) \rightarrow 0$ as $\eta \rightarrow 0$. Therefore, first letting $\epsilon \rightarrow 0$, and then $\eta \rightarrow 0$, we easily obtain

$$
\begin{aligned}
& 2^{-1} \int_{\Omega} \zeta^{6}\left|u_{x}(x, T)\right|^{2} \mathrm{~d} x+\int_{Q_{T} \cap\{u>0\}} \zeta^{6} u^{n} u_{x x x}^{2} \mathrm{~d} x \mathrm{~d} t \\
& \leqslant 2^{-1} \int_{\Omega} \zeta^{6}\left|u_{0 x}\right|^{2} \mathrm{~d} x-\int_{Q_{T} \cap\{u>0\}} u^{n} u_{x x x}\left[2 u_{x x}\left(\zeta^{6}\right)_{x}+u_{x}\left(\zeta^{6}\right)_{x x}\right] \mathrm{d} x \mathrm{~d} t \\
&-\int_{Q_{T} \cap\{u>0\}}\left(v u^{m} u_{x}-A u^{M} u_{x}\right)\left(u_{x} \zeta^{6}\right)_{x x} \mathrm{~d} x \mathrm{~d} t .
\end{aligned}
$$

Since $u(\cdot, t) \in \mathcal{C}^{1}(\bar{\Omega})$ for almost $t \in[0, T]$, it is possible to estimate from below the second term on the left hand side of (3.31) using the generalized Bernis inequalities given in Lemma 6.4. As a result we obtain

$$
\begin{aligned}
2^{-1} \int_{\Omega} \zeta^{6}\left|u_{x}(x, T)\right|^{2} \mathrm{~d} x+d_{7} \int_{Q_{T}} \zeta^{6}\left(\left(u^{(n+2) / 6}\right)_{x}^{6}+\left|\left(u^{(n+2) / 3}\right)_{x x}\right|^{3}+\left(u^{(n+2) / 2}\right)_{x x x}^{2}\right) \mathrm{d} x \mathrm{~d} t & +d_{9} \int_{Q_{T} \cap\{u>0\}} \zeta^{6} u^{n} u_{x x x}^{2} \mathrm{~d} x \mathrm{~d} t \\
\leqslant & 2^{-1} \int_{\Omega} \zeta^{6}\left|u_{0 x}(x)\right|^{2} \mathrm{~d} x+d_{10} \int_{Q_{T}}\left|\zeta_{x}\right|^{6} u^{n+2} \mathrm{~d} x \mathrm{~d} t \\
& -\int_{Q_{T} \cap\{u>0\}} u^{n} u_{x x x}\left[2 u_{x x}\left(\zeta^{6}\right)_{x}+u_{x}\left(\zeta^{6}\right)_{x x}\right] \mathrm{d} x \mathrm{~d} t \\
& -\int_{Q_{T} \cap\{u>0\}}\left(v u^{m} u_{x}-A u^{M} u_{x}\right)\left(u_{x} \zeta^{6}\right)_{x x} \mathrm{~d} x \mathrm{~d} t .
\end{aligned}
$$

Next we estimate the terms in the third integral on the right hand side as in [30]; namely,

$$
\begin{aligned}
\int_{Q_{T} \cap\{u>0\}} u^{n} u_{x x x} u_{x}\left(\zeta^{6}\right)_{x x} \mathrm{~d} x \mathrm{~d} t \\
\quad=6 \int_{Q_{T} \cap\{u>0\}}\left(u^{n / 2} u_{x x x} \zeta^{3}\right)\left(u^{(n-4) / 6} u_{x} \zeta\right)\left(u^{(n+2) / 3}\left(5 \zeta_{x}^{2}+\zeta \zeta_{x x}\right)\right) \mathrm{d} x \mathrm{~d} t
\end{aligned}
$$




$$
\begin{aligned}
& \leqslant 6\left(\int_{Q_{T} \cap\{u>0\}} u^{n} u_{x x x}^{2} \zeta^{6} \mathrm{~d} x \mathrm{~d} t\right)^{1 / 2}\left(\int_{Q_{T} \cap\{u>0\}} u^{n-4} u_{x}^{6} \zeta^{6} \mathrm{~d} x \mathrm{~d} t\right)^{1 / 6} \\
& \quad \times\left(\int_{Q_{T}} u^{n+2}\left(5 \zeta_{x}^{2}+\zeta\left|\zeta_{x x}\right|\right)^{3} \mathrm{~d} x \mathrm{~d} t\right)^{1 / 3} \\
& \leqslant \delta \int_{Q_{T} \cap\{u>0\}}\left(u^{n} u_{x x x}^{2}+\left(u^{(n+2) / 6}\right)_{x}^{6}\right) \zeta^{6} \mathrm{~d} x \mathrm{~d} t+c(\delta) \int_{Q_{T}} u^{n+2}\left(\zeta_{x}^{6}+\left(\zeta\left|\zeta_{x x}\right|\right)^{3}\right) \mathrm{d} x \mathrm{~d} t, \quad \forall \delta>0,
\end{aligned}
$$

and

$$
\begin{aligned}
& \int_{Q_{T} \cap\{u>0\}} u^{n} u_{x x x} u_{x x}\left(\zeta^{6}\right)_{x} \mathrm{~d} x \mathrm{~d} t \\
&=6 \int_{Q_{T} \cap\{u>0\}}\left(u^{\frac{n}{2}} u_{x x x} \zeta^{3}\right)\left[\left(u^{\frac{n-1}{3}} u_{x x}+\frac{n-1}{3} u^{\frac{n-4}{3}} u_{x}^{2}-\frac{n-1}{3} u^{\frac{n-4}{3}} u_{x}^{2}\right) \zeta^{2}\right]\left(u^{\frac{n}{2}-\frac{n-1}{3}} \zeta_{x}\right) \mathrm{d} x \mathrm{~d} t \\
& \leqslant 6\left(\int_{Q_{T} \cap\{u>0\}} u^{n} u_{x x x}^{2} \zeta^{6} \mathrm{~d} x \mathrm{~d} t\right)^{1 / 2} \\
& \times\left[\int_{Q_{T} \cap\{u>0\}}\left(\frac{3}{n+2}\left|\left(u^{\frac{n+2}{3}}\right)_{x x}\right|+\frac{n-1}{3}\left(\frac{6}{n+2}\right)^{2}\left(u^{\frac{n+2}{6}}\right)_{x}^{2}\right)^{3} \zeta^{6} \mathrm{~d} x \mathrm{~d} t\right]^{1 / 3} \\
& \times\left(\int_{Q_{T}} u^{n+2} \zeta_{x}^{6} \mathrm{~d} x \mathrm{~d} t\right)^{1 / 6} \\
& \leqslant \delta \int_{Q_{T} \cap\{u>0\}}\left(u^{n} u_{x x x}^{2}+\left|\left(u^{\frac{n+2}{3}}\right)_{x x}\right|^{3}+\left(u^{\frac{n+2}{6}}\right)_{x}^{6}\right) \zeta^{6} \mathrm{~d} x \mathrm{~d} t+c(\delta) \int_{Q_{T}} u^{n+2} \zeta_{x}^{6} \mathrm{~d} x \mathrm{~d} t, \quad \forall \delta>0 .
\end{aligned}
$$

Using these estimates in 3.32) with $\delta=d_{9} / 6$, we obtain 3.21.

\section{Finite speed propagation (strong slippage: $0<n<2$ )}

In this section, we consider problem $(\mathbb{P})$ with initial data, $u_{0}$, which satisfies 2.1 and which also possesses the additional property

$$
\operatorname{supp} u_{0} \subset\{x \leqslant 0\} .
$$

Let us introduce the following family of subdomains:

$$
\Omega(s)=\Omega \cap\{x \mid x>s\} \quad \forall s \in(-a, a), \quad Q_{T}(s)=\Omega(s) \times(0, T) .
$$

Theorem 5 Let $u_{0}$ satisfy (2.1), (4.1), let $v= \pm 1, A \geqslant 0,0<n<2, m<M$ if $A>0$, $m<n+2$ if $v=1, A=0$, and

$$
m>0 \quad \text { if } v=-1, \quad m>n / 2 \quad \text { if } v=1,
$$

and let $u$ denote an arbitrary strong nonnegative solution of problem $(\mathbb{P})$, obtained as in Theorem 1 , which satisfies the local entropy estimate in Theorem 2 or 3 . Then $u$ possesses the finite speed of propagation property in the sense that there exists a continuous function, $s(t)$, such that $s(0)=0$, and a positive time $T_{0}$, such that

$$
\operatorname{supp} u(\cdot, t) \subset \bar{\Omega} \backslash \Omega(s(t)), \quad s(t)<a \quad \forall t<T_{0}, \quad s\left(T_{0}\right)=a .
$$

REMARK 4.1 The analysis in this section also applies to (1.4)(a). 
Proof. Since by assumption $0<n<2$, the local entropy estimate, (3.17) or (3.18), holds for some positive $\alpha<2-n$. The proof of the finite speed of propagation property is based on a careful analysis of the properties of solutions satisfying these inequalities with positive $\alpha$. In the stable case, $v=-1$, such analysis was performed in [20] with $A=0$. If $v=-1$ and $A>0$, a similar proof can be given. Therefore we restrict our attention here to the unstable case, $v=1$. Although we set $A=0$ for simplicity, all our estimates are also valid for the case $v=1, A>0$. Thus, the estimate (3.18) can be written in the form

$$
\begin{array}{r}
\frac{1}{\alpha(\alpha+1)} \int_{\Omega} \zeta^{4} u^{1+\alpha}(x, T) \mathrm{d} x+c_{1} \int_{Q_{T}} \zeta^{4}\left(u^{\alpha+n-2 \gamma+1}\left(u^{\gamma}\right)_{x x}^{2}+u^{\alpha+n-3} u_{x}^{4}\right) \mathrm{d} x \mathrm{~d} t \\
\leqslant \frac{1}{\alpha(\alpha+1)} \int_{\Omega} \zeta^{4} u_{0}^{1+\alpha} \mathrm{d} x+c R, \quad \alpha>0,
\end{array}
$$

where $c:=\max \left(c_{2}, c_{3}, c_{4}\right)$ and

$$
\begin{aligned}
R:=R_{1}+R_{2}+R_{3}:= & \int_{Q_{T}}\left(\left|\zeta_{x}^{4}\right|+\left|\zeta \zeta_{x x}\right|^{2}\right) u^{n+\alpha+1} \mathrm{~d} x \mathrm{~d} t \\
& +\int_{Q_{T}}\left|\left(\zeta^{3} \zeta_{x}\right)_{x}\right| u^{\alpha+m+1} \mathrm{~d} x \mathrm{~d} t+\int_{Q_{T}} \zeta^{4} u^{\alpha+2 m-n+1} \mathrm{~d} x \mathrm{~d} t .
\end{aligned}
$$

Note now that due to the constraint $m>n / 2$, the minimal power of $u(x, t)$ in terms of the form $\int_{Q_{T}} \zeta^{4} u^{\sigma_{1}} \mathrm{~d} x \mathrm{~d} t$ on the right hand side of $\sqrt{4.4}$ is strictly greater than the minimal power of $u$ in terms of the form $\int_{\Omega} \zeta^{4} u^{\sigma_{2}} \mathrm{~d} x$ appearing on the left hand side of 4.4 ; namely,

$$
\alpha+2 m-n+1>\alpha+1 \Leftrightarrow m>n / 2 .
$$

This will allow us to derive a functional relationship from (4.4), for a suitable set of energy functions, which satisfies the conditions of the generalized Stampacchia Lemma, Lemma 6.2.

To put (4.4) in an appropriate form, we set $\zeta(x)=\zeta_{s, \delta}(x)$, where $\zeta_{s, \delta}(x)$ is the cut-off function defined as

$$
\zeta_{s, \delta}(x)=\varphi\left(\frac{x-s}{\delta}\right),
$$

where $s \in \mathbb{R}, \delta>0$ are free parameters, and $\varphi(r)$ is a nonnegative nondecreasing $\mathcal{C}^{2}(\mathbb{R})$ function such that

$$
\varphi(r)=0 \quad \text { for } r \leqslant 0, \quad \varphi(r)=1 \quad \text { for } r>1 .
$$

We now define three energy functions which are connected with the terms on the right hand side of our entropy estimate 4.4 ,

$$
\begin{aligned}
& J_{T}(s):=\int_{Q_{T}(s)} u^{\beta_{1}+\alpha+1} \mathrm{~d} x \mathrm{~d} t, \quad E_{T}(s):=\int_{Q_{T}(s)} u^{\beta_{2}+\alpha+1} \mathrm{~d} x \mathrm{~d} t, \\
& I_{T}(s):=\int_{Q_{T}(s)} u^{\beta_{3}+\alpha+1} \mathrm{~d} x \mathrm{~d} t,
\end{aligned}
$$

where $\beta_{1}=n, \beta_{2}=m, \beta_{3}=2 m-n$. Using (4.4), we shall deduce a system of three functional inequalities for $J_{T}(s), E_{T}(s), I_{T}(s)$. Setting $\zeta(x)=\zeta_{s, \delta}(x)$ in 4.4, we obtain after some simple computations 


$$
\begin{aligned}
\sup _{t \in(0, T)} \int_{\Omega(s+\delta)} & u^{\alpha+1} \mathrm{~d} x+D_{1} \int_{Q_{T}(s+\delta)}\left[\left(u^{(\alpha+n+1) / 2}\right)_{x x}^{2}+\left(u^{(\alpha+n+1) / 4}\right)_{x}^{4}\right] \mathrm{d} x \mathrm{~d} t \\
\leqslant & D_{2}\left[\frac{1}{\delta^{4}} \int_{Q_{T}(s) \backslash Q_{T}(s+\delta)} u^{\alpha+n+1} \mathrm{~d} x \mathrm{~d} t+\frac{1}{\delta^{2}} \int_{Q_{T}(s) \backslash Q_{T}(s+\delta)} u^{\alpha+m+1} \mathrm{~d} x \mathrm{~d} t\right. \\
& \left.+\int_{Q_{T}(s)} u^{\alpha+2 m-n+1} \mathrm{~d} x \mathrm{~d} t\right]+\int_{\Omega(s)} u_{0}^{1+\alpha} \mathrm{d} x .
\end{aligned}
$$

Here and throughout the proof, $D_{i}$ denote positive constants which can depend on the problem parameters, $\alpha, n, m$, but not on $s, \delta$, and $T$. For arbitrary $\beta>0, l>4, s>0, \delta>0$ such that $s+2 \delta<a$, we find for $\sigma=\beta+\alpha+1$ that

$$
\int_{\Omega(s+2 \delta)} u^{\sigma} \mathrm{d} x \leqslant \int_{\Omega(s+\delta)}\left(u \zeta_{s+\delta, \delta}^{l}\right)^{\sigma} \mathrm{d} x=\int_{\Omega(s+\delta)} v^{\frac{4 \sigma}{n+\alpha+1}} \mathrm{~d} x
$$

where $v=v(x, t)=\left(u \zeta_{s+\delta, \delta}^{l}\right)^{(n+\alpha+1) / 4}$. By the Gagliardo-Nirenberg interpolation inequality,

$$
\int_{\Omega(s+\delta)} v^{\frac{4 \sigma}{n+\alpha+1}} \mathrm{~d} x \leqslant D_{3}\left[\int_{\Omega(s+\delta)}\left|v_{x}\right|^{4} \mathrm{~d} x\right]^{\frac{\theta \sigma}{n+\alpha+1}}\left[\int_{\Omega(s+\delta)} v^{\frac{4(\alpha+1)}{n+\alpha+1}} \mathrm{~d} x\right]^{\frac{(1-\theta) \sigma}{\alpha+1}}
$$

where $\theta=\frac{\beta(n+\alpha+1)}{\sigma(n+4(\alpha+1))}$. Combining (4.9) and 4.10) gives

$$
\begin{aligned}
\int_{\Omega(s+2 \delta)} u^{\beta+\alpha+1} \mathrm{~d} x & \leqslant D_{4}\left[\int_{\Omega(s+\delta)} u^{\alpha+1} \mathrm{~d} x\right]^{\frac{(1-\theta) \sigma}{\alpha+1}} \\
\times & {\left[\int_{\Omega(s+\delta)}\left|\left(u^{(n+\alpha+1) / 4}\right)_{x}\right|^{4} \mathrm{~d} x+\frac{1}{\delta^{4}} \int_{\Omega(s+\delta) \backslash \Omega(s+2 \delta)} u^{n+\alpha+1} \mathrm{~d} x\right]^{\frac{\theta \sigma}{n+\alpha+1}} . }
\end{aligned}
$$

Let us suppose that

$$
\frac{\theta \sigma}{n+\alpha+1}<1
$$

or equivalently that

$$
\beta<n+4(\alpha+1) .
$$

Then, integrating 4.11 with respect to $t$ and using Hölder's inequality, we obtain

$$
\begin{gathered}
\int_{Q_{T}(s+2 \delta)} u^{\beta+\alpha+1} \mathrm{~d} x \mathrm{~d} t \leqslant D_{5} T^{1-\frac{\beta}{n+4(\alpha+1)}} \sup _{t \in(0, T)}\left(\int_{\Omega(s+\delta)} u^{\alpha+1} \mathrm{~d} x\right)^{\frac{(1-\theta)(\beta+\alpha+1)}{\alpha+1}} \\
\times\left[\frac{1}{\delta^{4}} \int_{Q_{T}(s+\delta) \backslash Q_{T}(s+2 \delta)} u^{n+\alpha+1} \mathrm{~d} x \mathrm{~d} t+\int_{Q_{T}(s+\delta)}\left|\left(u^{(n+\alpha+1) / 4}\right)_{x}\right|^{4} \mathrm{~d} x \mathrm{~d} t\right]^{\frac{\beta}{n+4(\alpha+1)}} \\
\leqslant D_{6} T^{1-\frac{\beta}{n+4(\alpha+1)}}\left[\sup _{t \in(0, T)} \int_{\Omega(s+\delta)} u^{\alpha+1} \mathrm{~d} x+\frac{1}{\delta^{4}} \int_{Q_{T}(s+\delta) \backslash Q_{T}(s+2 \delta)} u^{n+\alpha+1} \mathrm{~d} x \mathrm{~d} t\right. \\
\left.+\int_{Q_{T}(s+\delta)}\left|\left(u^{(n+\alpha+1) / 4}\right)_{x}\right|^{4} \mathrm{~d} x \mathrm{~d} t\right]^{1+\mu}
\end{gathered}
$$

where $\mu=4 \beta /(n+4(\alpha+1))>0$. 
Using the definitions in (4.7) and the a priori estimate (4.8), we deduce from (4.13) that

$$
\begin{aligned}
\int_{Q_{T}(s+2 \delta)} & u^{\beta+\alpha+1} \mathrm{~d} x \mathrm{~d} t \leqslant D_{7} T^{1-\mu / 4} \\
\times & \times\left[\frac{J_{T}(s)-J_{T}(s+2 \delta)}{\delta^{4}}+\frac{E_{T}(s)-E_{T}(s+\delta)}{\delta^{2}}+I_{T}(s)+\int_{\Omega(s)} u_{0}^{\alpha+1} \mathrm{~d} x\right]^{1+\mu} .
\end{aligned}
$$

The inequality (4.14) holds for the three values of $\beta_{i}, i=1,2,3$, prescribed in 4.7), if condition (4.12) holds with $\beta=\beta_{i}, i=1,2,3$. These conditions may be written as

$$
\text { 1) } \alpha+1>0, \quad 2) m<n+4(\alpha+1), \quad \text { 3) } 2 m-n<n+4(\alpha+1) \text {. }
$$

It is easy to check that all of these conditions are satisfied for some $\alpha \in\left(\alpha_{2}, 2-n\right)$ if and only if

$$
m<6-n .
$$

Thus, if the inequality 4.15 holds, we obtain the following system of functional inequalities:

$$
\begin{aligned}
J_{T}(s+\delta) \leqslant & D_{8} T^{\left(4-\mu_{1}\right) / 4} \\
& \times\left[\frac{J_{T}(s)-J_{T}(s+\delta)}{\delta^{4}}+\frac{E_{T}(s)-E_{T}(s+\delta)}{\delta^{2}}+I_{T}(s)+h_{0}(s)\right]^{1+\mu_{1}}, \\
E_{T}(s+\delta) \leqslant & D_{9} T^{\left(4-\mu_{2}\right) / 4} \\
& \times\left[\frac{J_{T}(s)-J_{T}(s+\delta)}{\delta^{4}}+\frac{E_{T}(s)-E_{T}(s+\delta)}{\delta^{2}}+I_{T}(s)+h_{0}(s)\right]^{1+\mu_{2}}, \\
I_{T}(s+\delta) \leqslant & D_{10} T^{\left(4-\mu_{3}\right) / 4} \\
& \times\left[\frac{J_{T}(s)-J_{T}(s+\delta)}{\delta^{4}}+\frac{E_{T}(s)-E_{T}(s+\delta)}{\delta^{2}}+I_{T}(s)+h_{0}(s)\right]^{1+\mu_{3}},
\end{aligned}
$$

where $h_{0}(s)=\int_{\Omega(s)} u_{0}^{1+\alpha} \mathrm{d} x$, and

$$
\mu_{1}=\frac{4 n}{n+4(\alpha+1)}, \quad \mu_{2}=\frac{4 m}{n+4(\alpha+1)}, \quad \mu_{3}=\frac{4(2 m-n)}{n+4(\alpha+1)} .
$$

Due to the boundedness and nonnegativity of $u$, the following estimates are obvious:

$$
\begin{array}{ll}
J_{T}(0) \leqslant J_{T}:=\int_{Q_{T}} u^{\beta_{1}+\alpha+1} \mathrm{~d} x \mathrm{~d} t<c T, & \forall T>0, \\
E_{T}(0) \leqslant E_{T}:=\int_{Q_{T}} u^{\beta_{2}+\alpha+1} \mathrm{~d} x \mathrm{~d} t<c T, & \forall T>0, \\
I_{T}(0) \leqslant I_{T}:=\int_{Q_{T}} u^{\beta_{3}+\alpha+1} \mathrm{~d} x \mathrm{~d} t<c T, & \forall T>0,
\end{array}
$$

where $c$ is a constant which does not depend on $T$. The validity of the statement of Theorem 5 when inequality 4.15) holds now follows from 4.16, 4.17), and Lemma 6.2, since $h_{0}(s)=0$ for any $s>0$. 
If $m \geqslant 6-n$, we proceed as follows. We fix $\bar{m}$ such that $n / 2<\bar{m}<6-n$. It is easy to see that due to the boundedness of the solution $u$, all of the previous estimates in the proof of Theorem 5 remain valid when $m$ is replaced by $\bar{m}$. As a result, the system 4.16 is obtained with respect to new energy functions 4.7 defined by the values

$$
\beta_{1}=n, \quad \beta_{2}=\bar{m}, \quad \beta_{3}=2 \bar{m}-m,
$$

and where

$$
\mu_{1}=\frac{4 n}{n+4(\alpha+1)}, \quad \mu_{2}=\frac{4 \bar{m}}{n+4(\alpha+1)}, \quad \mu_{3}=\frac{4(2 \bar{m}-n)}{n+4(\alpha+1)}
$$

In this manner, the validity of the statement of the theorem for the case $m \geqslant 6-n$ again follows from (4.16) and Lemma 6.2.

\section{Finite speed propagation (weak slippage: $2 \leqslant n<3$ )}

In this section we shall again consider problem $(\mathbb{P})$ with initial data $u_{0}$ which satisfies $(2.1)$ as well as the additional property 4.1). The subdomains $\Omega(s)$ and $Q_{t}(s)$ are to be understood here to be as defined in 4.2.

We first prove the following lemma, which provides control on the $L_{\text {loc }}^{1}(\Omega)$ norm of some minimal positive power of the solution under consideration, $u(x, t)$. For the sake of simplicity, the results in this section are proven for $v=1$ and $A=0$, though they remain valid for $v=-1$ and $A>0$ as well. The results here can also be readily shown to apply to 1.3 if $2<n<3$ and to (1.4)(a).

Lemma 5.1 Let $v= \pm 1, A \geqslant 0,1 / 2<n<3, m>n / 2, \eta>(1-n) / 3, \varepsilon>0$, with $m<M$ if $A>0$ and $m<n+2$ if $v=1, A=0$. Then there exists a positive constant $c$, depending on $n, m, \eta, \varepsilon$ only, such that any nonnegative strong solution $u$ of problem $(\mathbb{P})$ satisfies

$$
\begin{aligned}
\int_{\Omega} u(x, T)^{\eta+1} \zeta^{4} \mathrm{~d} x \leqslant & \varepsilon\left(\int_{Q_{T} \cap\{u>0\}} \zeta^{6} u^{n} u_{x x x}^{2} \mathrm{~d} x \mathrm{~d} t+\int_{Q_{T}}\left|\zeta_{x}\right|^{6} u^{n+2} \mathrm{~d} x \mathrm{~d} t\right) \\
& +c\left(\int_{Q_{T}}\left[u^{n+2 \eta}\left|\zeta_{x}\right|^{2}+u^{(3 m+3 \eta+1-n) / 2} \zeta^{3}+u^{m+\eta+1}\left|\zeta \zeta_{x}\right|^{2}\right] \mathrm{d} x \mathrm{~d} t\right) \\
& +\int_{\Omega} u_{0}^{\eta+1} \zeta^{4} \mathrm{~d} x+c \int_{Q_{T} \cap \operatorname{supp} \zeta} u^{n+3 \eta-1} \mathrm{~d} x \mathrm{~d} t
\end{aligned}
$$

for arbitrary nonnegative $\zeta \in \mathcal{C}^{2}([-a, a])$.

Proof. The proof here follows that of Lemma 5.2 in [25]. Let us set $\phi=\varphi$ in the integral identity in 2.2, where $\varphi$ is the test function

$$
\varphi=-l_{\delta} \zeta^{4}(u+\gamma)^{\eta}, \quad \gamma>0
$$


where $\left\{l_{\delta}\right\} \subset \mathcal{C}_{c}^{\infty}(0, T)$ and $l_{\delta} \rightarrow \chi_{(0, T)}$ as $\delta \rightarrow 0$. After some simple computations, we obtain

$$
\begin{aligned}
-\int_{Q_{T}}\left(l_{\delta}\right)_{t} \zeta^{4} \frac{(u+\gamma)^{\eta+1}}{\eta+1} \mathrm{~d} x \mathrm{~d} t= & \int_{Q_{T}} u^{m} u_{x} l_{\delta}\left((u+\gamma)^{\eta} \zeta^{4}\right)_{x} \mathrm{~d} x \mathrm{~d} t \\
& +\int_{Q_{T} \cap\{u>0\}} l_{\delta}\left(\zeta^{4}\right)_{x} u^{n}(u+\gamma)^{\eta} u_{x x x} \mathrm{~d} x \mathrm{~d} t \\
& +\eta \int_{Q_{T} \cap\{u>0\}} l_{\delta} \zeta^{4} u^{n}(u+\gamma)^{\eta-1} u_{x} u_{x x x} \mathrm{~d} x \mathrm{~d} t \\
= & A_{1}+A_{2}+A_{3} .
\end{aligned}
$$

The terms $A_{2}, A_{3}$ can be estimated as in [25, Lemma 5.2]. For any $\epsilon>0$,

$$
\begin{aligned}
\left|A_{2}\right| \leqslant & \epsilon \int_{Q_{T} \cap\{u>0\}} \zeta^{6} u^{n} u_{x x x}^{2} \mathrm{~d} x \mathrm{~d} t+C_{1}(\epsilon) \int_{Q_{T}}\left|\zeta_{x}\right|^{2} u^{n}(u+\gamma)^{2 \eta} \mathrm{d} x \mathrm{~d} t, \\
\left|A_{3}\right| \leqslant & \epsilon\left(\int_{Q_{T} \cap\{u>0\}} \zeta^{6} u^{n} u_{x x x}^{2} \mathrm{~d} x \mathrm{~d} t+\int_{Q_{T}}\left|\zeta_{x}\right|^{6} u^{n+2} \mathrm{~d} x \mathrm{~d} t\right) \\
& +C_{2}(\epsilon) \int_{Q_{T} \cap \operatorname{supp} \zeta}(u+\gamma)^{n+3 \eta-1} \mathrm{~d} x \mathrm{~d} t .
\end{aligned}
$$

Here $C_{i}$ denote constants which may depend on $m, n, \eta$, and on $\epsilon$ if indicated, but which are independent of $\gamma$ and $\delta$.

Let us now estimate $A_{1}$. We have

$$
A_{1}=\int_{Q_{T}} \eta u^{m}(u+\gamma)^{\eta-1} u_{x}^{2} \zeta^{4} l_{\delta} \mathrm{d} x \mathrm{~d} t+\int_{Q_{T}} 4 u^{m}(u+\gamma)^{\eta} u_{x} \zeta^{3} \zeta_{x} l_{\delta} \mathrm{d} x \mathrm{~d} t=: A_{1}^{(1)}+A_{1}^{(2)} .
$$

Since $m-\frac{n-4}{3}+\eta-1>0$, it follows from Young's inequality and Lemma 6.4 that for any $\epsilon>0$,

$$
\begin{aligned}
\left|A_{1}^{(1)}\right| \leqslant & \epsilon \int_{Q_{T} \cap\{u>0\}} u_{x}^{6} u^{n-4} \zeta^{6} \mathrm{~d} x \mathrm{~d} t+C_{3}(\epsilon) \int_{Q_{T}}(u+\gamma)^{\frac{3}{2}\left(m-\frac{n-4}{3}+\eta-1\right)} \zeta^{3} \mathrm{~d} x \mathrm{~d} t \\
\leqslant & \epsilon\left(\int_{Q_{T} \cap\{u>0\}} \zeta^{6} u^{n} u_{x x x}^{2} \mathrm{~d} x \mathrm{~d} t+C_{4} \int_{Q_{T}} \zeta_{x}^{6} u^{n+2} \mathrm{~d} x \mathrm{~d} t\right) \\
& +C_{3}(\epsilon) \int_{Q_{T}}(u+\gamma)^{(3 m+3 \eta+1-n) / 2} \zeta^{3} \mathrm{~d} x \mathrm{~d} t .
\end{aligned}
$$

With regard to $A_{1}^{(2)}$, we have by Young's inequality

$$
\left|A_{1}^{(2)}\right| \leqslant\left|A_{1}^{(1)}\right|+C_{5} \int_{Q_{T}}(u+\gamma)^{m+\eta+1}\left|\zeta \zeta_{x}\right|^{2} \mathrm{~d} x \mathrm{~d} t
$$

Thus all the integrals in 5.2 are uniformly bounded with respect to the parameters $\delta, \gamma>0$. Therefore, collecting the estimates obtained for the terms $A_{i}, i=1,2,3$, and passing to the limit $\delta \rightarrow 0$, and then $\gamma \rightarrow 0$, we obtain the estimate 5.1).

Theorem 6 Let $u_{0}$ satisfy 2.1], 4.1], let $v= \pm 1, A \geqslant 0,1 / 2<n<3, m>n / 2, m<M$ if $A>0$, and $m<n+2$ if $v=1, A=0$, and let $u$ denote an arbitrary strong nonnegative solution of problem $(\mathbb{P})$ obtained as in Theorem 1, which satisfies the local entropy estimate of Theorem 2 or 3 . Then $u$ possesses the finite speed of propagation property in the sense of Theorem 5 
Proof. Let us consider the local energy estimate (3.21) obtained in Theorem 4 , and estimate the third term on the right hand side, setting $v=1$ for simplicity:

$$
\begin{aligned}
B:= & \int_{Q_{T} \cap\{u>0\}} u^{m} u_{x}\left(u_{x} \zeta^{6}\right)_{x x} \mathrm{~d} x \mathrm{~d} t \\
= & \int_{Q_{T} \cap\{u>0\}} u^{m} u_{x} u_{x x x} \zeta^{6} \mathrm{~d} x \mathrm{~d} t+12 \int_{Q_{T} \cap\{u>0\}} u^{m} u_{x} u_{x x} \zeta^{5} \zeta_{x} \mathrm{~d} x \mathrm{~d} t \\
& +6 \int_{Q_{T} \cap\{u>0\}} u^{m} u_{x}^{2}\left(\zeta^{5} \zeta_{x}\right)_{x} \mathrm{~d} x \mathrm{~d} t=: B_{1}+B_{2}+B_{3}
\end{aligned}
$$

Using Young's inequality, we deduce that for any $\epsilon>0$,

$$
\begin{aligned}
\left|B_{1}\right| & \leqslant\left|\int_{Q_{T} \cap\{u>0\}} u^{n / 2} u_{x x x} u_{x} u^{(n-4) / 6} u^{m-n / 2-(n-4) / 6} \zeta^{6} \mathrm{~d} x \mathrm{~d} t\right| \\
& \leqslant \epsilon \int_{Q_{T} \cap\{u>0\}}\left(u^{n} u_{x x x}^{2}+u_{x}^{6} u^{n-4}\right) \zeta^{6} \mathrm{~d} x \mathrm{~d} t+D_{1}(\epsilon) \int_{Q_{T}} u^{3 m-2 n+2} \zeta^{6} \mathrm{~d} x \mathrm{~d} t .
\end{aligned}
$$

Here and below, $D_{i}$ denote constants which may depend on $m, n, \eta$, and on $\epsilon$ if indicated, but which are independent of $\delta$ and $s$. Similarly, we may estimate

$$
\begin{aligned}
& \left|B_{2}\right| \leqslant \epsilon \int_{Q_{T} \cap\{u>0\}}\left(\left|u_{x x}\right|^{3} u^{n-1}+u_{x}^{6} u^{n-4}\right) \zeta^{6} \mathrm{~d} x \mathrm{~d} t+D_{2}(\epsilon) \int_{Q_{T}} u^{2 m-n+2}\left|\zeta^{2} \zeta\right|^{2} \mathrm{~d} x \mathrm{~d} t, \\
& \left|B_{3}\right| \leqslant \epsilon \int_{Q_{T} \cap\{u>0\}} u_{x}^{6} u^{n-4} \zeta^{6} \mathrm{~d} x \mathrm{~d} t+D_{3}(\epsilon) \int_{Q_{T}} u^{(3 m-n+4) / 2}\left|\left(\zeta^{3} \zeta_{x}\right)_{x}\right|^{3 / 2} \mathrm{~d} x \mathrm{~d} t .
\end{aligned}
$$

Using these estimates in 3.21, we find that for $\epsilon>0$ sufficiently small,

$$
\begin{aligned}
\int_{\Omega}\left|u_{x}(x, T)\right|^{2} \zeta^{6} \mathrm{~d} x+\frac{d_{10}}{2} \int_{Q_{T}} \zeta^{6}\left[\left(u^{(n+2) / 6}\right)_{x}^{6}+\left|\left(u^{(n+2) / 3}\right)_{x x}\right|^{3}+\left(u^{(n+2) / 2}\right)_{x x x}^{2}\right] \mathrm{d} x \mathrm{~d} t & +\frac{d_{10}}{2} \int_{Q_{T} \cap\{u>0\}} \zeta^{6} u^{n} u_{x x x}^{2} \mathrm{~d} x \mathrm{~d} t \\
\leqslant & \int_{\Omega}\left|u_{0 x}\right|^{2} \zeta^{6} \mathrm{~d} x+d_{9} \int_{Q_{T}}\left(\left|\zeta_{x}\right|^{6}+\left|\zeta \zeta_{x x}\right|^{3}\right)|u|^{n+2} \mathrm{~d} x \mathrm{~d} t \\
& +D_{4} \int_{Q_{T}}\left[u^{3 m-2 n+2} \zeta^{6}+u^{2 m-n+2}\left|\zeta^{2} \zeta_{x}\right|^{2}+u^{(3 m-n+4) / 2}\left|\left(\zeta^{3} \zeta_{x}\right)_{x}\right|^{3 / 2}\right] \mathrm{d} x \mathrm{~d} t
\end{aligned}
$$

Assuming that $\eta>(1-n) / 3$, summing the inequalities [5.6, 5.1], and taking $\epsilon>0$ sufficiently small, we obtain

$$
\begin{aligned}
\int_{\Omega}|u(x, T)|^{\eta+1} \zeta^{4} \mathrm{~d} x+\int_{\Omega}\left|u_{x}(x, T)\right|^{2} \zeta^{6} \mathrm{~d} x & +\frac{d_{10}}{4} \int_{Q_{T} \cap\{u>0\}} \zeta^{6}\left|\left(u^{(n+2) / 2}\right)_{x x x}\right|^{2} \mathrm{~d} x \mathrm{~d} t \\
& \leqslant \int_{\Omega}\left|u_{0 x}\right|^{2} \zeta^{6} \mathrm{~d} x+\int_{\Omega}\left|u_{0}\right|^{\eta+1} \zeta^{4} \mathrm{~d} x+D_{5} R,
\end{aligned}
$$


where

$$
\begin{aligned}
R:= & \int_{Q_{T}} u^{3 m-2 n+2} \zeta^{6} \mathrm{~d} x \mathrm{~d} t+\int_{Q_{T}} u^{(3 m+3 \eta+1-n) / 2} \zeta^{3} \mathrm{~d} x \mathrm{~d} t \\
& +\int_{Q_{T} \cap \operatorname{supp} \zeta} u^{n+3 \eta-1} \mathrm{~d} x \mathrm{~d} t+\int_{Q_{T}}\left(\left|\zeta_{x}\right|^{6}+\left|\zeta \zeta_{x x}\right|^{3}\right) u^{n+2} \mathrm{~d} x \mathrm{~d} t \\
& +\int_{Q_{T}} u^{2 m-n+2}\left|\zeta^{2} \zeta_{x}\right|^{2} \mathrm{~d} x \mathrm{~d} t+\int_{Q_{T}} u^{n+2 \eta}\left|\zeta_{x}\right|^{2} \mathrm{~d} x \mathrm{~d} t \\
& +\int_{Q_{T}} u^{m+\eta+1}\left|\zeta \zeta_{x}\right|^{2} \mathrm{~d} x \mathrm{~d} t+\int_{Q_{T}} u^{(3 m-n+4) / 2}\left|\left(\zeta^{3} \zeta_{x}\right)_{x}\right|^{3 / 2} \mathrm{~d} x \mathrm{~d} t
\end{aligned}
$$

Let us take for $\zeta(x)$ the function $\zeta_{s, \delta}$ from 4.5 . It then follows from 5.7 that

$$
\begin{array}{r}
\sup _{t \in(0, T)} \int_{\Omega(s+\delta)}|u(x, t)|^{\eta+1} \mathrm{~d} x+\sup _{t \in(0, T)} \int_{\Omega(s+\delta)}\left|u_{x}(x, t)\right|^{2} \mathrm{~d} x+\frac{d_{10}}{4} \int_{Q_{T}(s+\delta)}\left|\left(u^{(n+2) / 2}\right)_{x x x}\right|^{2} \mathrm{~d} x \mathrm{~d} t \\
\leqslant \int_{\Omega(s)}\left(\left|u_{0 x}\right|^{2} \mathrm{~d} x+\left|u_{0}\right|^{\eta+1}\right) \mathrm{d} x+D_{6} \tilde{R}
\end{array}
$$

where

$$
\begin{aligned}
\tilde{R}:= & \int_{Q_{T}(s)} u^{3 m-3 n+2} \mathrm{~d} x \mathrm{~d} t+\int_{Q_{T}(s)} u^{(3 m+3 \eta+1-n) / 2} \mathrm{~d} x \mathrm{~d} t+\int_{Q_{T}(s)} u^{n+3 \eta-1} \mathrm{~d} x \mathrm{~d} t \\
& +\delta^{-6} \int_{Q_{T}(s)} u^{n+2} \mathrm{~d} x \mathrm{~d} t+\delta^{-2} \int_{Q_{T}(s)} u^{2 m-n+2} \mathrm{~d} x \mathrm{~d} t \\
& +\delta^{-2} \int_{Q_{T}(s)} u^{n+2 \eta} \mathrm{d} x \mathrm{~d} t+\delta^{-2} \int_{Q_{T}(s)} u^{m+\eta+1} \mathrm{~d} x \mathrm{~d} t+\delta^{-3} \int_{Q_{T}(s)} u^{(3 m-n+4) / 2} \mathrm{~d} x \mathrm{~d} t \\
=: & \sum_{i=1}^{8} \delta^{-\chi_{i}} \int_{Q_{T}(s)} u^{\xi_{i}} \mathrm{~d} x \mathrm{~d} t .
\end{aligned}
$$

We shall use the inequality (5.8) to derive a system of functional relationships which will allow us to implement the Stampacchia Lemma, Lemma 6.2. In order to undertake similar computations to those in $\S 4$, it suffices to guarantee that

$$
\xi_{i}>1+\eta, \quad i=1, \ldots, 8
$$

First we ensure that

$$
\xi_{3}=n+3 \eta-1>1+\eta \Leftrightarrow \eta>1-n / 2=: \eta_{\min } .
$$

Next we deduce a restriction on $m$ by considering

$$
\xi_{1}=3 m-2 n+2>1+\eta \Leftrightarrow \frac{3}{2}(2 m-n)+1+(1-n / 2)>1+\eta .
$$

Together, 5.10 and 5.11 yield

$$
\eta_{\min }=1-n / 2<\eta<\eta_{\min }+\frac{3}{2}(2 m-n) .
$$


There exists $\eta$ satisfying $[5.12]$ iff

$$
2 m-n>0
$$

Next it is easy to see that

$$
\xi_{2}=\frac{3 m+3 \eta+1-n}{2}=\frac{(n+3 \eta-1)+(3 m-2 n+2)}{2} .
$$

Therefore the inequality

$$
\xi_{2}>1+\eta
$$

follows from (5.10) and (5.11). It is easy to check that the other inequalities in (5.9) can be satisfied by an appropriate choice of $\eta$ if conditions (5.10) and (5.11) are satisfied.

As a result of 5.9], the following Gagliardo-Nirenberg interpolation inequalities hold for $i=$ $1, \ldots, 8$ :

$$
\begin{aligned}
\int_{\Omega(s+2 \delta)} u^{\xi_{i}} \mathrm{~d} x \leqslant & D_{7}\left(\int_{\Omega(s+\delta)}\left|\left(\left(\zeta_{s+\delta, \delta}^{6 /(n+2)} u\right)^{(n+2) / 2}\right)_{x x x}\right|^{2} \mathrm{~d} x\right)^{\theta_{i} \xi_{i} /(n+2)} \\
& \times\left(\int_{\Omega(s+\delta)}\left(\zeta_{s+\delta, \delta}^{6 /(n+2)} u\right)^{\eta+1} \mathrm{~d} x\right)^{\frac{\left(1-\theta_{i}\right) \xi_{i}}{\eta+1}}
\end{aligned}
$$

where $\theta_{i}=\frac{(n+2)\left(\xi_{i}-\eta-1\right)}{\xi_{i}(5 \eta+n+7)}, i=1, \ldots, 8$. We now wish to guarantee that

$$
\frac{\theta_{i} \xi_{i}}{n+2}<1, \quad i=1, \ldots, 8
$$

From the definition of $\theta_{i}$, it follows that 5.16 holds iff

$$
\eta>\frac{\xi_{i}-n-8}{6}, \quad i=1, \ldots, 8
$$

It is easy to check that all the inequalities in 5.9 and 5.17 hold for some $\eta$ in the interval 5.12 if condition (5.13) is satisfied.

Therefore we deduce from (5.8), (5.15) the following inequalities:

$$
\int_{Q_{T}(s+\delta)} u^{\xi_{i}} \mathrm{~d} x \mathrm{~d} t \leqslant D_{8} T^{1-\frac{\theta_{i} \xi_{i}}{n+2}}\left(\sum_{j=1}^{8} \delta^{-\chi_{j}} \int_{Q_{T}(s)} u^{\xi_{j}} \mathrm{~d} x \mathrm{~d} t+H_{0}(s)\right)^{1+\frac{6\left(\xi_{i}-\eta-1\right)}{5 \eta+n+7}}
$$

where $H_{0}(s):=\int_{\Omega(s)}\left(u_{0}^{1+\eta}+\left|u_{0 x}\right|^{2}\right) \mathrm{d} x=0$ for all $0<s<s+\delta<a$. From 5.18) and Lemma 6.2 , the conclusion of Theorem 6 now follows.

REMARK 5.2 The method used here for the qualitative analysis of conditions which guarantee the finite speed of propagation property should be possible to modify, modulo various technical difficulties, to yield quantitative predictions for propagation rates, [25, 30, 45]. We hope to perform such an analysis in a forthcoming paper. 


\section{Appendix A}

LEMMA 6.1 (Stampacchia's Lemma [48]) Assume that a given nonnegative nonincreasing function $g:[0, \infty) \rightarrow[0, \infty)$ satisfies

$$
g(s+\delta) \leqslant c_{0}\left(\delta^{-\alpha} g(s)\right)^{\beta}, \quad \forall s, \delta>0,
$$

for some real numbers $c_{0}>0, \alpha>0, \beta>1$. Then

$$
g\left(s_{0}\right)=0, \quad s_{0}:=2^{\beta /(\beta-1)}\left(c_{0} g(0)^{\beta-1}\right)^{1 / 2 \beta} .
$$

For given $\left(b_{1}, \ldots, b_{k}\right) \in \mathbb{R}^{k}$, we denote

$$
b=\prod_{j=1}^{k} b_{j}, \quad \bar{b}_{i}=\frac{b}{b_{i}}=\prod_{j=1, j \neq i}^{k} b_{j} .
$$

LEMma 6.2 (A Stampacchia Lemma for Systems [25]) Assume that $k$ given nonnegative nonincreasing functions $g_{i}:[0, \infty) \rightarrow[0, \infty), i=1, \ldots, k$, satisfy

$$
g_{i}(s+\delta) \leqslant c_{i}\left(\sum_{i=1}^{k} \delta^{-\alpha_{i}} g_{i}(s)\right)^{\beta_{i}}, \quad \forall s, \delta>0, i=1, \ldots, k,
$$

for some real numbers $c_{i}>0, \beta_{i}>1, \alpha_{i} \geqslant 0, i=1, \ldots, k$, where $\alpha_{i}>0$ for $i=1, \ldots, l$, for some $l$ with $1 \leqslant l \leqslant k$. Denote

$$
g(s)=\sum_{i=1}^{k} \overline{c_{i}^{\bar{\beta}_{i}}} g_{i}(s)^{\bar{\beta}_{i}},
$$

and assume that the function $Q(s)$ defined by

$$
Q(s)= \begin{cases}\left.k^{\beta} \sum_{i=l+1}^{k} c_{i}^{\bar{\beta}_{i}} \overline{\left(c_{i}^{\bar{\beta}_{i}}\right.}\right)^{1-\beta_{i}} g(s)^{\beta_{i}-1} & \text { if } l<k, \\ 0 & \text { if } l=k,\end{cases}
$$

satisfies the constraint $Q\left(s_{1}\right)<1$ at some point $s_{1} \geqslant 0$. Then there exists a positive constant $C>1$, which depends on $k, l, \alpha_{i}, \beta_{i}$, and $Q\left(s_{1}\right)$, such that

$$
g_{i}\left(s_{0}\right)=0, \quad \forall i=1, \ldots, l
$$

where

$$
\left.s_{0}:=s_{1}+C \sum_{i=1}^{l}\left(c_{i}^{\bar{\beta}_{i}} \overline{\left(c_{i}^{\bar{\beta}_{i}}\right.}\right)^{1-\beta_{i}} g\left(s_{1}\right)^{\beta_{i}-1}\right)^{\frac{1}{\alpha_{i} \beta}} .
$$

REMARK 6.3 If $l=k$, then $s_{1}=0$ in Lemma 6.2

LEMMA 6.4 (Generalized Bernis inequalities for periodic functions [25]) Let $\Omega=(a, b) \subset \mathbb{R}^{1}$, $n \in(1 / 2,3)$, and let $u \in \mathcal{C}^{1}(\bar{\Omega}) \cap H_{\text {loc }}^{3}(\{u>0\})$ be an arbitrary $|\Omega|$-periodic function such that

$$
u \geqslant 0, \quad u_{x} \in L^{2}(\Omega), \quad \int_{\{u>0\}} u^{n}\left|u_{x x x}\right|^{2} \mathrm{~d} x<\infty .
$$


Then $u^{(n+2) / 6} \in W^{1,6}(\Omega), u^{(n+2) / 3} \in W^{2,3}(\Omega), u^{(n+2) / 2} \in W^{3,2}(\Omega)=: H^{3}(\Omega)$, and there exists a positive constant, $C>1$, which depends on $n$ only, such that

$$
\begin{aligned}
\int_{\Omega} \zeta^{6}\left(\left|\left(u^{(n+2) / 6}\right)_{x}\right|^{6}+\left|\left(u^{(n+2) / 3}\right)_{x x}\right|^{3}\right. & \left.+\left|\left(u^{(n+2) / 2}\right)_{x x x}\right|^{2}\right) \mathrm{d} x \\
& \leqslant C \int_{\{u>0\}} \zeta^{6} u^{n}\left|u_{x x x}\right|^{2} \mathrm{~d} x+C \int_{\Omega}\left|\zeta_{x}\right|^{6} u^{n+2} \mathrm{~d} x
\end{aligned}
$$

where $\zeta(x)$ is an arbitrary $\mathcal{C}^{1}$ nonnegative $|\Omega|$-periodic function.

\section{Acknowledgments}

A. N.-C. would like to acknowledge the support of the M.R. Saulson Research Grant. A. Sh. would like to acknowledge the hospitality and support of the Math Department of the Technion during visits to Haifa, Israel.

\section{REFERENCES}

1. Beretta, E. Selfsimilar source solutions of a fourth order degenerate parabolic equation. Nonlinear Anal. 29 (1997), 741-760. Zbl 0879.35083 MR 1455063

2. Beretta, E., Bertsch, M., \& Dal PAsso, R. Nonnegative solutions of a fourth-order nonlinear degenerate parabolic equation. Arch. Ration. Mech. Anal. 129 (1995), 175-200. Zbl 0827.35065 MR 1328475

3. BERNIS, F. Nonlinear parabolic equations arising in semiconductor and viscous droplet models. In: Nonlinear Diffusion Equations and Their Equilibrium States, 3 (Gregynog, 1989), W.-M. Ni et al. (eds.), Birkhäuser (1992), 77-88. Zbl 0793.35107 MR 1167830

4. BERnis, F. Finite speed of propagation and continuity of the interface for thin viscous flows. $A d v$. Differential Equations 1 (1996), 337-368. Zbl 0846.35058 MR 1401398

5. BERnis, F. Finite speed of propagation for thin viscous flows when $2 \leqslant n<3$. C. R. Acad. Sci. Paris Sér. I Math. 322 (1996), 1169-1174. Zbl 0853.76018 MR 1396660

6. BERNIS, F. Integral inequalities with application to nonlinear degenerate parabolic equations. In: Nonlinear Problems in Applied Mathematics, T. S. Angell et al. (eds.), SIAM, Philadelphia (1996), 57-65. Zbl 0889.35054 MR 2410598

7. Bernis, F., \& Friedman, A. Higher order nonlinear degenerate parabolic equations. J. Differential Equations 83 (1990), 179-206. Zbl 0702.35143 MR 1031383

8. Bernis, F., Peletier, L. A., \& Williams, S. M. Source type solutions of a fourth order nonlinear degenerate parabolic equation. Nonlinear Anal. 18 (1992), 217-234. Zbl 0778.35056 MR 1148286

9. Bertozzi, A. L., Brenner, M. P., Dupont, T. F., \& Kadanoff, L. P. Singularities and similarities in interface flows. In: Trends and Perspectives in Applied Mathematics, Appl. Math. Sci. 100, L. Sirovich (ed.), Springer (1994), 155-208. Zbl 0808.76022 MR 1277195

10. Bertozzi, A. L., Grün, G., \& Witelski, T. P. Dewetting films: bifurcations and concentrations. Nonlinearity 14 (2001), 1569-1592. Zbl 1006.35049 MR 1867093

11. Bertozzi, A. L., \& Pugh, M. The lubrication approximation for thin viscous films: the moving contact line with a porous media cutoff of the van der Waals interactions. Nonlinearity 7 (1994), 1535-1564. Zbl 0811.35045 MR 1304438

12. Bertozzi, A. L., \& PUGH, M. Lubrication approximation for thin viscous films: regularity and long time behavior of weak solutions. Comm. Pure Appl. Math. 49 (1996), 85-123. Zbl 0863.76017 MR 1371925 
13. Bertozzi, A. L., \& Pugh, M. Long-wave instabilities and saturation in thin film equations. Comm. Pure Appl. Math. 51 (1998), 625-661. Zbl 0916.35008 MR 1611136

14. Bertozzi, A. L., \& Pugh, M. Finite time blow-up of solutions of some long-wave unstable thin film equations. Indiana Univ. Math. J. 49 (2000), 1323-1366. Zbl 0978.35007 MR 1836532

15. Bertsch, M., Dal Passo, R., Garcke, H., \& Grün, G. The thin viscous flow equation in higher space dimensions. Adv. Differential Equations 4 (1998), 417-440. Zbl 0954.35035 MR 1751951

16. Bonn, D., Eggers, J., Indekeu, J., Meunier, J., \& Rolley, E. Wetting and spreading. Rev. Modern Phys. 81 (2009), 739-805.

17. Dal Passo, R., \& Garcke, H. Solutions of a fourth order degenerate parabolic equation with weak initial trace. Ann. Scuola Norm. Sup. Pisa Cl. Sci. 28 (1999), 153-181. Zbl 0945.35049 MR 1679081

18. DAl PASSO, R., GARCKe, H., \& GRÜN, G. On a fourth-order degerate parabolic equation: global entropy estimates, existence, and qualitative behavior of solutions. SIAM J. Math. Anal. 29 (1998), 321342. Zbl 0929.35061 MR 1616558

19. Dal Passo, R., Giacomelli, L., \& GRÜN, G. A waiting time phenomenon for thin film equations. Ann. Scuola Norm. Sup. Pisa Cl. Sci. 30 (2001), 437-463. Zbl 1024.35051 MR 1895718

20. Dal Passo, R., Giacomelli, L., \& Shishkov, A. The thin film equation with nonlinear diffusion. Comm. Partial Differential Equations 26 (2001), 1509-1557. Zbl 1001.35070 MR 1865938

21. Ehrhard, P., \& DAVIS, S.H. Non-isothermal spreading of liquid drops on horizontal plates. J. Fluid Mech. 229 (1991), 365-388. Zbl 0850.76760

22. Eidel'man, S. D. Parabolic Systems. North-Holland, Amsterdam (1969). Zbl 0181.37403 MR 0252806

23. Elliott, C. M., \& GARCKe, H. On the Cahn-Hilliard equation with degenerate mobility. SIAM J. Math. Anal. 27 (1996), 404-423. Zbl 0856.35071 MR 1377481

24. Giacomelli, L., A fourth-order degenerate parabolic equation describing thin viscous flows over an inclined plane. Appl. Math. Lett. 12 (1999), 107-111. Zbl 0964.35084 MR 1751343

25. Giacomelli, L., \& Shishrov, A. Propagation of support in one-dimensional convected thin-film flow. Indiana Univ. Math. J. 54 (2005), 1181-1215. Z Zbl 1088.35050 MR 2164423

26. GRÜN, G., Degnerate parabolic differential equations of fourth order and a plasticity model with non-local hardening. Z. Anal. Anwendungen 14 (1995), 541-574. Zbl 0835.35061 MR 1362530

27. GRÜN, G. On the convergence of entropy consistent schemes for lubrication type equations in multiple space dimensions. Math. Comput. 72 (2003), 1251-1279. Zbl 1084.65093 MR 1972735

28. GRÜN, G., \& RUMPF, M. Nonnegativity preserving convergent schemes for the thin film equation. Numer. Math. 87 (2000), 113-152. Zbl 0988.76056 MR 1800156

29. Hocherman, T., \& Rosenau, P. On KS-type equations describing the evolution and rupture of a liquid interface. Phys. D 67 (1993), 113-125. Zbl 0787.76092

30. Hulshof, J., \& Shishrov, A. The thin film equation with $2 \leqslant n<3$ : finite speed of propagation in terms of the $L^{1}$-norm. Adv. Differential Equations 3 (1998), 625-642. Zbl 0953.35072 MR 1665858

31. IsRaelachVili, J. N. Intermolecular and Surface Forces. 2nd ed., Academic Press, London (1991).

32. Kersner, R., \& Shishrov, A. Existence of free boundaries in thin-film theory. Preprint (1996).

33. Klapper, I., \& Dockery, J. Role of cohesion in the material description of biofilms. Phys. Rev. E 74 (2006), 031902-1-031902-8.

34. Laugesen, R. S., \& Pugh, M. C. Properties of steady states for thin film equations. Eur. J. Appl. Math. 11 (2000), 293-351. Zbl 1041.76008 MR 1844589

35. Laugesen, R. S., \& PUgh, M. C. Linear stability for steady states of thin film equations. Arch. Ration. Mech. Anal. 154 (2000), 3-51. Zbl 0980.35030 MR 1778120

36. Laugesen, R. S., \& Pugh, M. C. Energy levels of steady states for thin film type equations. J. Differential Equations 182 (2002), 377-415. Zbl 1011.34005 MR 1900328 
37. Laugesen, R. S., \& Pugh, M. C. Heteroclinic orbits, mobility parameters and stability for thin film type equations. Electron. J. Differential Equations 2002, no. 95, 29 pp. Zbl 1029.35121 MR 1938391

38. Minkov, E., \& Novick-Cohen, A. Droplet profiles under the influence of van der Waals forces. Eur. J. Appl. Math. 12 (2001), 367-393; Errata, ibid. 17 (2006), 128. Zbl 1097.76552 MR 2228974

39. Novick-Cohen, A. Energy methods for the Cahn-Hilliard equation. Quart. Appl. Math. 46 (1988), 681-690. Zbl 0685.35050 MR 0973383

40. Novick-Cohen, A. The Cahn-Hilliard equation. In: Handbook of Differential Equations. IV Evolutionary Partial Differential Equations, C. Dafermos and M. Pokorny (eds.), Elsevier (2008) 201228. Zbl 1185.35001 MR 2508166

41. Oron, A., Davis, S. H., \& BAnKoff, S. G. Long-scale evolution of thin liquid films. Rev. Modern Phys. 69 (1997), 931-980.

42. Oron, A., \& Rosenau, P. Formation of patterns induced by thermocapillarity and gravity. J. Phys. (France) II 2 (1992), 131-146.

43. Отто, F. Lubrication approximation with prescribed non-zero contact angle. Comm. Partial Differential Equations 23 (1998), 2077-2164. Zbl 0923.35211 MR 1662172

44. Sharma, A., \& Ruckenstein, E. An analytical nonlinear theory of thin film rupture and its application to wetting films. J. Colloid Interface Sci. 113 (1986), 456-479.

45. Shishkov, A. E. Waiting time of propagation and the backward motion of interfaces in thin-film flow theory. Discrete Contin. Dynam. Systems 2007, suppl., 938-945. Zb1 1163.35405 MR 2409931

46. SLEPČEV, D. Linear stability of selfsimilar solutions of unstable thin-film equations. Interfaces Free Bound. 11 (2009), 375-398. Zbl 1180.35432 MR 2546604

47. SlePČEV, D., \& PUGH, M.C. Self-similar blowup of unstable thin-film equations. Indiana Univ. Math. J. 54 (2005), 1697-1738. Zbl 1091.35071 MR 2189683

48. Stampacchia, G. Équations elliptiques du second ordre à coefficients discontinus. Sém. Math. Sup. 16, Presses Univ. Montréal, Montréal (1966). Zbl 0151.15501 MR 0251373

49. Thiele, U., \& Knobloch, E. Thin liquid films on a slightly inclined plate. Phys. D 190 (2004), 213248. Zbl 1063.76032 MR 2043346

50. Williams, M., \& Davis, S. H. The non-linear theory of film rupture. J. Colloid Interface Sci. 90 (1982), 220-228.

51. Witelski, T. P., \& Bernoff, A. J. Dynamics of three-dimensional thin film rupture. Phys. D 147 (2000), 155-176. Zbl 0992.76013 MR 1793238

52. Witelski, T. P., Bernoff, A. J., \& Bertozzi, A. L. Blowup and dissipation in a critical-case unstable thin film equation. Eur. J. Appl. Math. 15 (2004), 223-256. Zbl 1062.76005 MR 2069680

53. Zhang, W. W., \& Lister, J. R. Similarity solutions for van der Waals rupture of a thin film on a solid substrate. Phys. Fluids 11 (1999), 2454-2462. Z Zbl 1149.76597| MR 1707811 